\title{
Stichwort: Implementationsforschung zwischen Intervention und Transfer im Kontext von Mehrsprachigkeit und sprachlicher Bildung
}

\author{
Hans-Joachim Roth • Yasemin Uçan · Sonja Sieger • Christina Gollan
}

Eingegangen: 16. Oktober 2020 / Überarbeitet: 25. Februar 2021 / Angenommen: 20. Mai 2021 / Online publiziert: 23. Juni 2021

(C) Der/die Autor(en) 2021

Zusammenfassung Im vorliegenden Beitrag wird ausgehend von der Unterscheidung in einen weiten und einen engen Implementationsbegriff nach Schrader et al. (2020) ein Überblick über den Stand der Implementationsforschung zu sprachlicher Bildung mit einem Schwerpunkt auf Mehrsprachigkeit gegeben. Dafür wird zunächst eine Systematik zum Verständnis von Implementationsforschung vorgelegt, an die sich eine Darstellung der zwei großen bundesweiten Programme im Feld der sprachlichen Bildung in Deutschland - FörMig und BiSS - anschließt, um den Entwicklungsprozess der Forschung zu Implementation von sprachlichen Bildungsmaßnahmen nachzuzeichnen. Im Anschluss werden weitere aktuelle Studien zur Sprachbildung und -förderung im Elementarbereich gesichtet und - als exemplarischer Blick auf die internationale Forschung zur Implementation mehrsprachiger Bildung - Ergebnisse zum Ansatz des Translanguaging präsentiert. Der Beitrag schließt mit einer Standortbestimmung der aktuellen Implementationsforschung zu

Prof. Dr. Hans-Joachim Roth ( $₫)$

Humanwissenschaftliche Fakultät, Department Erziehungs- und Sozialwissenschaften, Universität zu Köln, Gronewaldstr. 2, 50931 Köln, Deutschland

E-Mail: hans-joachim.roth@uni-koeln.de

Mercator-Institut für Sprachförderung und Deutsch als Zweitsprache, Universität zu Köln, Triforum I Albertus-Magnus-Platz, 50923 Köln, Deutschland

Yasemin Uçan · Sonja Sieger · Dr. Christina Gollan

Abteilung Sprache und Bildungssystem, Mercator-Institut für Sprachförderung und Deutsch als

Zweitsprache, Universität zu Köln, Triforum I Albertus-Magnus-Platz, 50923 Köln, Deutschland

Yasemin Uçan

E-Mail: yasemin.ucan@mercator.uni-koeln.de

Sonja Sieger

E-Mail: sonja.sieger@mercator.uni-koeln.de

Dr. Christina Gollan

E-Mail: christina.gollan@mercator.uni-koeln.de 
sprachlicher Bildung und Mehrsprachigkeit anhand von zusammenfassenden Thesen zu Anlage und Gelingensbedingungen von Implementation in der sprachlichen Bildung.

Schlüsselwörter Sprachliche Bildung · Mehrsprachigkeit · Translanguaging · Implementation · Transfer · Intervention

\title{
Keyword: Implementation research between intervention and transfer in the context of multilingualism and language education
}

\begin{abstract}
Starting from the distinction between a broad and a narrow implementation concept according to Schrader et al. (2020), this article gives an overview of the state of implementation research on language education with a focus on multilingualism. To this end, a systematic approach to understanding implementation research will be presented first, followed by a description of the two large nationwide programmes in the field of language education in Germany-FörMig and BiSS - in order to trace the development process of research into the implementation of language education measures. Subsequently, further current studies on language education and promotion at elementary level are reviewed and-as an exemplary view of international research on the implementation of multilingual education-results on the approach of translanguaging will be presented. The article concludes with a summary of current implementation research on language education and multilingualism on the basis of recapitulatory theses on the structure and conditions for success of implementation in language education.
\end{abstract}

Keywords Language education · Multilingualism · Translanguaging · Implementation · Transfer · Intervention

Wie Schiffer sind wir, die ihr Schiff auf offener See umbauen müssen, ohne es jemals in einem Dock zerlegen und aus besten Bestandteilen neu errichten zu können. (Otto Neurath 1981 [1932])

\section{Einleitung: Implementation und Transfer sprachlicher Bildung - ein diverses Feld}

Implementationsforschung im Kontext sprachlicher Bildung ist ein noch junges und diverses Feld. Wie Schrader et al. (2020) in ihrem Stichwortbeitrag zur Implementationsforschung im Bildungswesen in dieser Zeitschrift gezeigt haben, liegen in der Erziehungswissenschaft kaum Studien vor, die dem von den Autor*innen formulierten Standard der „Implementationsforschung im engeren Sinne“ - nämlich der Beforschung von bereits hinsichtlich ihrer Wirksamkeit geprüften Intervention entsprechen. Letztlich konnten die Autor*innen 33 Studien in ihren systematischen Literaturüberblick einbeziehen (Schrader et al. 2020, S. 22), von denen nur zwei dem Kontext sprachlicher Bildung zuzurechnen sind. Für die weitergreifende Frage 
des Transfers von Programmen sprachlicher Bildung gibt es keine den Suchkriterien entsprechenden Untersuchungen.

Ausgehend von dieser ersten Bestandsaufnahme stellen sich zwei Fragen: Zum einen ist danach zu fragen, was überhaupt unter Implementation und Transfer in der sprachlichen Bildung - welche Ergebnisse zu einsprachig wie mehrsprachig angelegten Modelle umfasst - zu verstehen ist; zum anderen bleibt offen, welche Faktoren und Elemente in Implementationsprozessen zu berücksichtigen sind. Denn wenn man den Blick weiter schweifen lässt, erscheint das Wissen doch reicher, als man anhand des o.g. Befundes annehmen kann. Betrachtet man nämlich das Thema nicht nur aus der Sicht des deutschen Bildungssystems mit seiner inzwischen doch sehr hohen Aufmerksamkeit auf Fragen der sprachlichen Bildung, sondern berücksichtigt auch internationaler Perspektiven, ergeben sich - gerade beim Thema des Umgangs mit mehrsprachigen Programmen - ganz grundsätzliche Implementationsfragen, die weit weg von der Ebene des konkreten Unterrichts liegen. So lokalisiert Benson (2004) in einem Beitrag zu zwei Fallstudien zur Implementation bilingualen Unterrichts in Mosambik und Bolivien das Problem nicht im Mangel wissenschaftlicher Erkenntnisse, sondern auf der Ebene bildungspolitischer Diskurse und Entscheidungsinstanzen. Dabei wird deutlich, dass in den unterschiedlichen Bildungssystemen der Transfer eines Programms mehrsprachigen Unterrichts, das die jeweils lokalen Sprachen in den Bildungsprozess einbezieht und deutlich klare und positive Wirkungen erzeugt, nicht gelingt. Als Hauptproblem für den Transfer macht Benson die mangelnde Akzeptanz verschiedener Akteursgruppen aus der Bildungspolitik und Lehrkräfteverbänden aus und stellt auch in einem aktuellen Beitrag fest, dass die vor 15 Jahren beschriebenen Probleme bis heute fortbestehen (vgl. Wong und Benson 2019, S. 8).

Vor dem Hintergrund dieser Herausforderungen von Implementationsforschung auf den Ebenen der Wirksamkeit, der Reichweite, der Akzeptanz und der Akteur*innen konzentriert sich dieser Überblicksbeitrag auf Erkenntnisse zur Implementation von Konzepten und Maßnahmen sprachlicher Bildung und schließt dazu an das Verständnis von Implementationsforschung im weiteren Sinne (vgl. Schrader et al. 2020) an. Es werden dementsprechend ebenfalls Studien einbezogen, die Konzepte untersuchen, welche bislang keine Wirksamkeitsprüfung durchlaufen haben sowie eine Reihe von Interventionsstudien, die am Rande auch Fragen der Implementation berücksichtigen. Gleichzeitig geht es darum, die zurzeit keineswegs abgeschlossene Entwicklung des Themas Implementation als einen Prozess in den Blick zu nehmen. Dazu wird anhand von Programmen der sprachlichen Bildung die Emergenz des Themenfelds aus einer partiell subjektiven Perspektive bis hin zur Entfaltung einer eigenständigen Implementationsforschung nachverfolgt, die bislang noch eng an Evaluations- und Interventionsstudien gekoppelt ist, sich aber aktuell als eigenständige Forschungsrichtung in der sprachlichen Bildung abzuzeichnen beginnt. Dieser Prozess wird als Überblick auf zwei Ebenen präsentiert: zum einen hinsichtlich übergreifender implementationstheoretischer Fragestellungen zu Akzeptanz, Konzepttreue, Praktikabilität, Verlauf und der Rolle verschiedener Akteur*innen. Zum anderen werden aus den ausgewerteten Studien konkrete Elemente sprachlicher Bildung extrahiert, die für Implementationsprozesse von Bedeutung sind. Die meisten der recherchierten Studien stammen aus dem deutschen Kontext 
und beziehen sich auf die Implementation von Maßnahmen und Programmen, die sich auf die deutsche Sprache konzentrieren. Zielgruppen sind ein- und mehrsprachige Schüler*innen sowie Kinder im Elementarbereich. In diesen Maßnahmen und Programmen spielt die Mehrsprachigkeit eine Rolle, allerdings ist diese als spezifische Bedingung des Lernens der deutschen Sprache berücksichtigt, nicht als ein konstitutives Element. Studien, die die lebensweltliche Mehrsprachigkeit als eigenen Bildungsgegenstand berücksichtigen, sind deutlich seltener; sie werden im Folgenden anhand von Studien zum bilingualen Unterricht sowie der - im Rahmen einer international angelegten Recherche gewonnenen - Studien zum neueren Ansatz des Translanguaging einbezogen.

Um einen Überblick über den aktuellen und auch internationalen Forschungsstand zur Implementationsforschung zu sprachlicher Bildung zu gewinnen, wurden zwei Rechercheschritte unternommen: Zum einen wurden Veröffentlichungen zu bundesweiten Programmen anhand der Veröffentlichungen der beteiligten Forscher*innen gezielt gesucht, zum anderen wurde eine Literaturrecherche anhand von Suchbegriffen durchgeführt. Für diese Recherche wurden folgende Datenbanken genutzt: Fachportal Pädagogik, Premier, ERIC und Google Scholar über die direkte Abfrage, sowie PsychINFO, PSYNDEX und Academic Search über eine Sammelabfrage via EBSCOhost. Es wurden Publikationen gesucht, die die folgenden Suchbegriffe enthielten: Transfer, Multiplikation, Fortbildung, Implementation, Implementationsforschung, Evaluationsforschung, Bedingungen, Gelingensbedingungen, Professionalisierung, Qualitätsentwicklung, Qualitätssicherung. Um die Suchergebnisse zu spezifizieren und auf das entsprechende Forschungsfeld zu reduzieren, um welches es in diesem Beitrag gehen soll, wurden die Suchbegriffe jeweils mit den Begriffen „sprachliche Bildung“, „Mehrsprachigkeit“ und „Translanguaging“ kombiniert. Außerdem wurden auch englisch- sowie spanischsprachige Äquivalente für die Suchanfragen genutzt. Die erzielten Ergebnisse wurden anhand der Abstracts auf ihre Passung geprüft, wobei hier eher weiche Ausschlusskriterien angelegt und alle Ergebnisse berücksichtigt wurden, die im weitesten Sinne mit der Intervention, Implementation oder Konzeptualisierung sprachlicher Bildungsmaßnahmen zu tun hatten, um nicht zu früh die - ohnehin schon wenigen Publikationen - weiter zu reduzieren und den Blick auf das Feld zu stark einzuschränken. Insgesamt wurden 32 Studien einbezogen (s. die Übersicht im Anhang).

Zum Verständnis der Strukturierung des folgenden Beitrags nehmen wir ein Ergebnis vorweg: So zeigte sich eine starke Streuung hinsichtlich der thematischen Breite und der Menge der Forschungsarbeiten in den einzelnen Themenfeldern (dual language education, language heritage programs, revitalization), die keine systematische Zusammenschau zulässt. Wir konzentrieren uns daher auf die Konzepte sprachlicher Bildung, zu denen eine hinreichende Menge von Studien für einen Überblick vorliegen.

Im Folgenden wird zunächst das Verhältnis von Implementationsforschung zu Interventionsforschung und Transferforschung vorgestellt (Abschn. 2). Daran anschließend wird die Entwicklung hin zu einer Implementationsforschung mit Fokus auf den Umgang mit Mehrsprachigkeit beschrieben (Abschn. 3); im Anschluss werden Ergebnisse aus zwei bundesweiten Programmen zur Implementation sprachlicher Bildung vorgestellt (Abschn. 4). Darauf folgt ein Überblick zum Translanguaging 
als Konzept mehrsprachiger Bildung (Abschn. 5). Der Beitrag schließt mit einer thesenartigen Zusammenfassung der Befunde (Abschn. 6).

\section{Intervention, Implementation und Transfer - Versuch einer Abgrenzung}

Für ein besseres Verständnis von Implementationsforschung im Verhältnis zu Interventions- und Transferforschung wird zunächst eine Art idealtypischer Prozess skizziert, den eine Innovation im Bildungssystem durchläuft, welcher in der Realität jedoch durch Überschneidungen und fließende Übergänge gekennzeichnet ist. Diesem idealtypischen Verständnis nach befassen sich die drei Forschungsgebiete mit verschiedenen Phasen der Umsetzung von Innovationen in einem bestehenden (Bildungs-)System, nämlich (1) mit der Entwicklung und Prüfung einer Bildungsmaßnahme (Interventionsforschung), (2) ihrer Umsetzung und Passung in verschiedene Kontexte (Implementationsforschung) und (3) ihrer flächendeckenden Verbreitung (Transferforschung).

Den Ausgangspunkt für die Umsetzung von Innovationen im Bildungssystem bildet im Idealfall eine Bestandsaufnahme (Diagnostik), um die bestehenden Probleme zu identifizieren. Je nach Ergebnis werden gezielte Maßnahmen (Interventionen) entwickelt, die die für nötig erachteten Veränderungen im System bewirken sollen. Interventionen können dabei auf individueller Ebene durchgeführt werden (MikroEbene), auf der Ebene von Verbünden, Organisationen und Institutionen (MesoEbene) oder auf System-Ebene (Makro-Ebene) (vgl. Leutner 2013, S. 18). Ziel der Interventionsforschung ist die Prüfung der Wirksamkeit einer Intervention während bzw. nach ihrer Umsetzung mit Hilfe wissenschaftlicher Methoden. Diese Prüfung findet, um kausale Interpretationen zuzulassen und damit beobachtete Veränderungen zuverlässig auf die umgesetzte Maßnahme zurückführen zu können, unter kontrollierten Bedingungen statt (vgl. Leutner 2013; Schrader et al. 2020, S. 16). Dabei haben sich im Bereich der quantitativen empirischen Forschung - auch für pädagogische Kontexte - randomisierte Kontrollstudien als methodischer „Goldstandard“ etabliert (Leutner 2013), wobei dies die Nutzung anderer methodischer Ansätze wie Vergleichsstudien und Korrelationsanalysen nicht ausschließt (Bellmann und Müller 2011). Neben einer Betrachtung der intendierten Wirkungen wird dafür plädiert, auch mögliche unerwünschte Nebenwirkungen im Rahmen einer Interventionsstudie zu berücksichtigen (Brügelmann 2019).

Nachdem die Wirksamkeit einer Intervention in einer kontrollierten Umgebung fundiert belegt wurde, schließt im idealtypischen Fall der Implementationsprozess und eine Identifikation der institutionellen, organisationalen und personalen Bedingungen an, welche für die erfolgreiche Umsetzung der Intervention in unterschiedlichen, nicht kontrollierten Kontexten nötig sind (vgl. Schrader et al. 2020, S. 16). ${ }^{1}$ Dies ist das fundamentale Erkenntnisinteresse der Implementationsforschung. Eine

\footnotetext{
1 Souvignier \& Philipp (2016, S. 10) bestimmen Erfolg in Anlehnung an das Modell von Proctor et al. (2009) nach sieben Kriterien: Akzeptanz, Angemessenheit, Machbarkeit, Wiedergabetreue, Implementationskosten, Durchdringung und Nachhaltigkeit.
} 
Implementationsforschung im engeren Sinne folgt nach Schrader et al. (2020) der oben skizzierten idealtypischen Abfolge und betrachtet nur auf ihre Wirksamkeit geprüfte Interventionen. Häufig findet man jedoch In Implementationsforschung im weiteren Sinne vor, die auch empirisch ungeprüfte Maßnahmen einschließt.

Diese Beobachtung entspricht dem Wesen verschiedener Implementationsstrategien, wie Gräsel und Parchmann (2004) sie definieren. Die Autorinnen unterscheiden zwischen drei Typen: Bottom-up-Strategien, Top-down-Strategien und symbiotischen Strategien. Im ersten Fall erwachsen die umzusetzende Intervention und die dazugehörigen Ziele, Methoden und Erfolgskriterien aus der Praxis, im zweiten Fall werden sie von außen vorgegeben. Als maßgeblich für eine Erfolgsbewertung des Implementationsprozesses gilt die möglichst getreue Umsetzung dieser Planungen in die Praxis. Während dieser Implementationsansatz sich gut mit dem Verständnis von Implementationsforschung im engeren Sinne deckt, korrespondieren symbiotische Implementationsstrategien mit einem stark partizipativen Zuschnitt eher mit einer Implementationsforschung im weiteren Sinne. Auch in diesem Ansatz wird die umzusetzende Maßnahme nicht von außen vorgegeben, sondern gemeinsam von den verschiedenen Akteur*innen - ausgehend von einer gemeinsam identifizierten und als veränderungswürdig erachteten Problemlage - erarbeitet. Es werden keine vorgefertigten Maßnahmen umgesetzt und dann die notwendigen Kontextbedingungen identifiziert, sondern Maßnahmenentwicklung und Kontextualisierung finden parallel statt und beeinflussen sich wechselseitig (Gräsel und Parchmann 2004; siehe auch Meyers et al. 2012; Fixsen et al. 2005 für einen stärkeren Fokus auf Implementation als Prozess). Erfolg wird im Rahmen von symbiotischen Implementationsstrategien dementsprechend nicht als eine möglichst getreue Umsetzung der geplanten Intervention verstanden. Stattdessen wird eine Implementation dann als erfolgreich eingestuft, wenn es ihr gelingt, Kooperationsstrukturen zu etablieren, die beschriebene problematische Ausgangslage zu verbessern oder zu beheben und schließlich die Intervention und ihre Aktivitäten weiter zu verbreiten.

An diesem Punkt finden sich fließende Übergänge zur Transferforschung, die im idealtypischen Ablauf an die Erkenntnisse der Implementationsforschung anschließt. Sie untersucht die Verbreitung von Innovationen und, wie der Übergang von einzelnen, gezielten Umsetzungen einer Maßnahme in eine flächendeckende Nutzung erfolgreich gestaltet werden kann (vgl. Gräsel 2010, S. 8 ff.; Schrader et al. 2020, S. 15). Anders als in der Implementationsforschung liegt der Fokus auf dem Handeln der Anbieter*innen einer Intervention und weniger auf der Seite der Nutzenden (Schrader et al. 2020, S. 15). Auch in diesem Forschungsbereich werden Erfolgsfaktoren und verschiedene Transferstrategien im Bildungsbereich untersucht und beschrieben (siehe z. B. Gräsel 2010, S. 11 ff.). Dabei erkennt die Forschung an, dass sowohl Implementations- als auch Transferprozesse nicht ohne Konsequenzen für die ursprüngliche Intervention bleiben. Es finden Selektionen und Umdeutungen statt, die Intervention wird also von den betroffenen Akteur*innen nicht einfach übernommen, sondern sozusagen neu erfunden (Rolff 2019, S. 53 ff.; Schrader et al. 2020, S. 19).

Die Trennschärfe zwischen den drei Forschungsgebieten ist - wie oben erwähnt nicht immer gegeben. So behandeln Interventionsstudien auch den Einfluss von Kontextfaktoren, Implementationsstudien betrachten ungeprüfte Interventionen und 
Implementationsstudien bedienen sich der Systematisierung von Erfolgsfaktoren aus der Transferforschung (siehe z.B. Hartmann et al. 2020).

Legt man die vorgestellte Systematik an den Bereich der sprachlichen Bildung an, so zeigt sich - wie eingangs genannt -, dass kaum Studien bestehen, die sich der Implementationsforschung im engeren Sinne zuordnen lassen. Ausgehend von einer Implementationsforschung im weiteren Sinne nach Schrader et al. (2020) zeigt sich, dass im Feld der sprachlichen Bildung wissenschaftlich abgesicherte Wirkungsanalysen vor allem für eng geschnittene Trainingskonzepte vorliegen - hingegen kaum für weiter ausgreifende Unterrichts- oder Schulentwicklungskonzepte. Hierzu existieren zwar evaluative Studien wie zum Beispiel zur interkulturellen Schulentwicklung im Hinblick auf den Umgang mit sprachlicher Bildung und Mehrsprachigkeit (vgl. Fürstenau 2016; Gomolla et al. 2016) oder Studien zur immersiven Beschulung in bilingualen Modellen (vgl. Duarte 2011; Gantefort 2013; Möller et al. 2016) sowie zu mehrsprachigen Elternbildungsprogrammen wie Rucksack (vgl. Lengyel et al. 2019; Roth und Terhart 2015). Allerdings geben diese häufig keine Auskunft über die Wirkung einzelner Maßnahmen im Detail, sondern sind so angelegt, dass über den Einsatz von Evaluationsinstrumenten unterschiedlicher Art - seien sie testförmig oder rekonstruktiv-interpretativ - Effekte der untersuchten Konzepte eher holistisch erfasst werden, ohne eindeutig und kausal auf konkrete Bedingungen ihres „Einbaus“ zurückgeführt werden zu können.

Für die folgenden Überlegungen ist grundsätzlich zu berücksichtigen, dass Implementation ein sehr komplexes Feld ist, das alle Ebenen von der Entscheidungsfindung, der Steuerung, der Adaption, der Qualifizierung, der direkt und mittelbar Beteiligten, der Didaktik, des Materials, der institutionellen Rahmenbedingungen und auch der gesellschaftlichen Diskurse umfasst. Implementationsforschung, die all diese Ebenen methodisch abgesichert zu berücksichtigen versucht, kann letztlich nur in großen und interdisziplinär zusammengesetzten Forschungsverbünden erfolgen. Diese Art von Forschung steht in der sprachlichen Bildung am Anfang. Wie hat sich das im Bereich der sprachlichen Bildung entwickelt? Wir beginnen mit den noch tentativen Erfahrungen in einem älteren Schulversuch.

\section{Zur Emergenz der Vorstellung der Implementation mehrsprachiger Bildung im deutschen Bildungssystem - eine Erinnerung}

Ende der 1990er-Jahre beschloss die Hamburger Schulbehörde als erste in Deutschland die Einsetzung eines Schulversuchs „Bilinguale Grundschulklassen“, um eine zweisprachige Schulbildung im Primarbereich anzubieten, die dem gesellschaftlichen Wandel einer dauerhaften Einwanderung im Bereich des Bildungswesens entgegenkam. Es ging dabei nicht um die frühe Einbeziehung schulischer Fremdsprachen, sondern um einen bilingualen Unterricht nach kanadischem und US-amerikanischem Vorbild, der die lebensweltliche Mehrsprachigkeit in die Schule hineinholte. Es wurden Klassen eingerichtet, in denen sowohl zweisprachige wie einsprachig deutsche Kinder in den Sprachenpaaren Italienisch-Deutsch, Spanisch-Deutsch, Portugiesisch-Deutsch und später auch Türkisch-Deutsch unterrichtet wurden. Organisatorisch wurde das Modell der two-way immersion gewählt (vgl. Reich und Roth 
2002) nach einer Verteilung von 50:50. Es wurden Klassen aus ungefähr gleich großen Gruppen von Schüler*innen aus einsprachig deutschen und mehrsprachigen Familien gebildet. Auch der Unterricht in den beiden Sprachen verteilte sich nach diesem Modell; der übrige Unterricht fand bis auf Ausnahmen in der deutschen Sprache statt. In den USA firmiert dieses Modell nach einer kritischen Diskussion zum Ausdruck ,,bilingual“ seit einigen Jahren unter dem Namen dual language education (vgl. Lindholm-Leary 2012, 2018).

Da zu dieser Zeit in Deutschland kaum wissenschaftliche Studien zur Auswirkung eines bilingualen Unterricht vorhanden waren und auch in der internationalen Forschung keine validen und unstrittigen Befunde vorlagen, wurde mit der Einsetzung des Schulversuchs entschieden, eine wissenschaftliche Begleitung zu installieren. Diese sollte die zunächst vier, später sechs beteiligten Grundschulen hinsichtlich der Entwicklung der Zweisprachigkeit bis in die Klassenstufe sechs hinein untersuchen. Die über die Jahre gewonnenen Ergebnisse liegen in Forschungsberichten an die auftraggebende Schulbehörde der Stadt Hamburg (vgl. u. a. Gogolin et al. 2007)² sowie einzelnen Veröffentlichungen vor (vgl. Duarte 2011; Gogolin und Roth 2007; Neumann und Roth 2004, 2009).

Nach den methodisch gut abgesicherten Ergebnissen der Studie zu den EuropaSchulen in Berlin (vgl. Möller et al. 2016) kann der grundsätzliche Optimismus, dass zweisprachiger Unterricht nicht nur für zweisprachig aufwachsende Schüler*innen das Lernen in der Schule bereichert und verbessert, auf der Wirkungsebene als hinreichend belegt gelten - insbesondere für Schüler*innen aus Migrationsfamilien, die zuhause die Sprachen ihrer Herkunft verwenden. Für die Frage, wie das Hamburger Modell implementiert wurde und welche Elemente sich dabei als besonders wichtig erwiesen haben, ist zunächst festzuhalten, dass der Begriff Implementation seinerzeit noch gar nicht zur Verfügung stand. Es fand also eine Implementation statt, ohne dass der Prozess als solcher so bezeichnet worden wäre. Man sprach in dieser Zeit eher von „Umsetzung“ - der Ausdruck lässt gut erkennen, wie wenig komplex der Prozess eingeschätzt wurde, der inzwischen als Implementation bezeichnet wird. Das erscheint nicht untypisch für Implementationsprozesse in einer Zeit, in der man daran ging, ein Modell aus einem anderen Bildungskontext - in diesem Fall Kanada, die USA, Schweden und einige wenige andere Länder - in ein anderes Bildungssystem zu transferieren, ohne die dafür nötige Adaption zu berücksichtigen. Man operierte, ohne hinreichende empirische Belege dafür zu haben, dass das entsprechende Modell überhaupt wirken konnte und ohne ein Wissen davon, unter welchen Bedingungen und in welchen Kontexten eine positive Wirkung überhaupt möglich sei. Auslöser war das Problem der geringeren Bildungserfolge mehrsprachiger Schüler*innen und das Brachliegen ihrer sprachlichen Ressourcen - eine soziale Dringlichkeit, die Implementationen im Bildungsbereich häufig motiviert (vgl. Schrader et al. 2020, S. 17).

Die Stadt Hamburg hatte 1999 den Auftrag an die wissenschaftliche Begleitung vergeben, die Entwicklung der Zweisprachigkeit zu untersuchen, um der damals mit Heftigkeit geführten Debatte gegen den bilingualen Unterricht (vgl. Gogolin

\footnotetext{
${ }^{2}$ Die Berichte waren stets im Internet zugänglich, vgl. etwa https://www.ew.uni-hamburg.de/ueber-diefakultaet/personen/gogolin/pdf-dokumente/bericht2007.pdf.
} 
und Neumann 2009) entgegentreten zu können. In den Schulen war diese Debatte auf allen Ebenen lebendig, vorrangig aber fanden sich die beteiligten Wissenschaftler*innen als Implementationsbegleiter in der Praxis wieder. Es war eben im wahrsten Sinne des Wortes ein „Schulversuch“. Das umfasste Elternabende, auf denen sich interessierte Eltern erkundigen konnten, wie sie sich den Unterricht vorstellen sollten, aber vor allem, welche Vorteile oder Nachteile für ihre Kinder entstehen könnten. Damals konnte man den Eltern lediglich mit einer gewissen Sicherheit sagen, dass Untersuchungen in anderen Ländern keine Nachteile, insbesondere für die einsprachigen Schüler*innen, erbracht hätten, und - mit einer gewissen Vorsicht - Vorteile für die Sprachentwicklung insgesamt zu erwarten seien (vgl. hierzu Reich und Roth 2002). Des Weiteren ging es darum, die Lehrkräfte in ihren Entscheidungen zur Organisation des Unterrichts zu unterstützen. Im Laufe der wissenschaftlichen Begleitung entwickelte sich eine dichte Arbeitsatmosphäre und Kommunikation zwischen den beteiligten Lehrkräften, Wissenschaftler*innen sowie der Schulbehörde, begleitet von regelmäßig stattfindenden Workshops, in denen Teilergebnisse vorgestellt und diskutiert wurden. Die wissenschaftliche Begleitung hatte für die Beteiligten ein Stadium der Nähe erreicht, das ein vertrauensvolles Verhältnis ermöglichte, ebenso das Aushandeln durchaus unterschiedlicher Erwartungen und Ansprüche, eine partizipative Implementationsstrategie mit deutlich symbiotischen Zügen, wie es Gräsel und Parchmann (2004) nennen. Das Projektteam wurde durch diese Erfahrungen also erst auf das Thema Implementation und seine Bedeutung gestoßen, wie gesagt, ohne bereits einen eigenen Begriff davon zu haben.

Das soll an einem Beispiel der bereits genannten Organisationsform des bilingualen Unterrichts verdeutlicht werden. Es war vorgesehen, dass die Kinder im Hamburger Modell von Anfang an nicht nach Sprachherkunft getrennt im Deutschen und in der Partnersprache unterrichtet werden sollten, wie es in den Berliner Europaschulen in den ersten zwei Jahren vorgesehen ist (vgl. Zydatiß 2000), sondern in sprachheterogenen Gruppen. Aufgrund fehlender Regelungen nutzen die beteiligten Lehrkräfte den Freiraum, um die Zusammensetzung der Klassen nach eigenen Vorstellungen zu gestalten. Daraus ergab sich eine unterschiedliche Organisation der ersten vier bilingualen Klassen, die jeweils ein eigenes Organisationsmodell verwendeten. Dieses Thema kam bereits am Anfang des Schulmodells im Jahr 2000 auf, weil die Lehrkräfte wie auch die Schulleitungen unsicher waren, wie die Umsetzung am besten funktionieren könnte. Die Frage wurde schnell an die wissenschaftliche Begleitung herangetragen und dominierte die ersten gemeinsamen Besprechungen. Da es keine verlässlichen empirischen Befunde gab, kam man überein, dass die Lehrkraft-Tandems selbst entscheiden sollten, wie sie verfahren und dass diese Entscheidung als ein Element in die Beobachtung der Sprachentwicklung der Lerngruppen eingehen sollte. Im Ergebnis wurden zwei Modelle mit unterschiedlichen Mischungsverhältnissen erprobt, nämlich (1.) zwei getrennte, aber hinsichtlich der Sprachherkunft gemischte Gruppen für den Sprachunterricht in Deutsch und der Partnersprache mit stabiler Zusammensetzung der Schüler*innen und (2.) durchgehendes Teamteaching der Sprachlehrkräfte im Sprachunterricht mit nur gelegentlichen funktional begründeten Gruppentrennungen in unterschiedlichen Zusammensetzungen.

In beiden Modellen entwickelten sich die Sprachkompetenzen aller Kinder im Deutschen gut. Der aus der IGLU-Studie zur Verfügung gestellte Lesekompetenz- 
test am Ende des vierten Schuljahrs zeigte Ergebnisse, die alle in der Nähe des für Deutschlands gebildeten Mittelwerts lagen (Gogolin et al. 2007, S. 144ff.). Die Auswertung der grammatischen Kompetenzen in der Mündlichkeit erbrachte vom ersten bis zum vierten Schuljahr ganz deutliche Zuwächse (Gogolin et al. 2007, S. 48 ff.). Hinsichtlich bildungssprachlicher Phänomene - auch ein Konzept, für das es damals noch keinen Begriff gab - ließ allein die Gruppe der Kinder, die ganz ohne oder mit nur geringen Deutschkenntnissen ins erste Schuljahr gekommen war, eine signifikant weniger fortgeschrittene Entwicklung erkennen. Alle anderen zweisprachigen Kinder unterschieden sich statistisch nicht von den einsprachig deutsch eingeschulten Kindern. Diese Gruppe der einsprachig deutsch eingeschulten Kinder erwies sich hingegen in den Partnersprachen als deutlich weniger weit entwickelt als alle anderen (Gogolin et al. 2007, S. 49 ff.). Dieses Ergebnis erscheint durchaus erwartbar, da die Dauer der Entwicklung einer ausgeglichenen Zweisprachigkeit nach Lage der internationalen Forschung zwischen fünf und sieben Jahren braucht (vgl. Reich und Roth 2002, S. 35). In der Folge wurde der Schulversuch in die Sekundarstufe hinein weitergeführt, um den Schüler*innen die Möglichkeit zu geben, die in der Grundschule erfolgreich angelegte Entwicklung ihrer Sprachkompetenzen weiterzuführen. Für die Gruppe der portugiesisch-deutsch unterrichteten Schüler*innen liegt eine Studie vor, die die durchweg positiven Ergebnisse darstellt (Duarte 2011).

Für den Hamburger Schulversuch am Ende der Grundschulzeit konnte aufgrund der erhobenen Daten zum sozioökonomischen Status der Familien außerdem gezeigt werden, dass diese Art des Unterrichts - insbesondere in den im Teamteaching unterrichteten Klassen - die Möglichkeit bietet, die im deutschen Schulsystem starke Abhängigkeit schulischer Leistungen in der deutschen Sprache vom ökonomischen und kulturellen Kapital der Familien partiell zu entkoppeln (vgl. Gogolin et al. 2007, S. $147 \mathrm{f}$.).

Für den vorliegenden Zusammenhang der Implementation lässt sich somit festhalten, dass nicht allein die Übernahme eines Unterrichtsmodells wie hier der twoway immersion betrachtet werden kann, sondern dass auch die Organisation der Differenzierung nach Sprachgruppen eine wesentliche Variable darstellt. Insofern gibt es nicht ein bilinguales Modell, sondern verschiedene. Über den oben genannten Erfahrungsaustausch sind die Schulen und Lehrkräfte alle dazu übergegangen, Teile des Unterrichts im Team zu erteilen. Dies hatte auch den Vorteil, dass auf diese Art und Weise der Sprachunterricht enger an den Sachunterricht gekoppelt werden und die sprachliche Bearbeitung sachunterrichtlicher Gegenstände sprachintegriert erfolgen konnte. Implementation gestaltete sich als ein Prozess, der damit bei weitem nicht abgeschlossen war, sondern über die gesamte Schulversuchszeit hinweg andauerte, weil jedes neue Schuljahr neue Herausforderungen bereithielt und Adaptionen nötig machte. Im Laufe der Zeit rückte somit die kontinuierliche Entwicklung der Passung des Modells mit den Bedingungen eines auf Mehrsprachigkeit nicht eingestellten Schulsystems in den Vordergrund der wissenschaftlichen Begleitung. Das lässt sich treffend mit der diesem Text als Motto vorangestellten Metapher Otto Neuraths vom Umbau des Schiffs auf offener See zusammenfassen.

Wie hat sich das Themenfeld entwickelt? Einige bundesweite Programme aus den letzten beiden Jahrzehnten erlauben weitere Einblicke in Forschungszugänge 
und Prozesse sowie Ergebnisse zu Fragen von Wirksamkeit, Organisation und Erfolgskriterien für die Implementation sprachlicher Bildung.

\section{Implementationsrelevante Erkenntnisse aus bundesweiten Programmen und Maßnahmen mit einem Ausblick auf den Elementarbereich}

\subsection{Beiträge zu Fragen von Implementation und Transfer}

Betrachtet man nun das Feld der sprachlichen Bildung etwas breiter und nicht nur die - zumindest in Deutschland - vergleichsweise wenige Schüler*innen betreffende bilingualen Modelle, so lässt sich Folgendes festhalten: Programme zur Verbesserung der Bildungssituation von zugewanderten Kindern und Jugendlichen haben eine bemerkenswerte Geschichte. Bereits in den siebziger und achtziger Jahren des letzten Jahrhunderts gab es 85 Modellversuche (vgl. Gogolin et al. 2011, S. 13). Die beiden letzten großen bundesweiten Programme - Förderung von Kindern und Jugendlichen mit Migrationshintergrund (FörMig) (2004-2009) und Bildung durch Sprache und Schrift (BiSS) (2013-2020) - waren also nicht die neue Entdeckung eines Themas, sondern stehen in einer Tradition der Auseinandersetzung mit Fragen der Bildungsbe(nach)teiligung von zugewanderten Kindern und Jugendlichen. Allerdings markieren sie nicht nur aufgrund ihres Umfangs eine Veränderung der Perspektive. Große Auswirkungen auf Fragen von Implementation und Transfer hatte nämlich die in den beiden genannten Programmen im Laufe des Prozesses vollzogene Abkehr von der Fokussierung auf Kinder „mit Migrationshintergrund“. Mit der in FörMig formulierten Perspektive einer ,durchgängigen Sprachbildung“ mit Schwerpunkt auf der Bildungssprache als das Sprachregister, in dem institutionelle Bildung vorrangig agiert und das wesentlich über den Bildungserfolg mit entscheidet, wurde die Blickrichtung von den betroffenen Schüler*innen und ihrer Herkunft weg und auf die sprachliche Bildung und ihre institutionelle Organisation verschoben. Gegenstand der Bemühungen war und ist somit nicht mehr eine wie auch immer definierte Gruppe von Schüler*innen, sondern die sprachpädagogische Aktivität selbst. Und diese wurde darüber für alle Schüler*innen geöffnet, abhängig von deren Bildungsbedarfen und nicht von Herkunft und/oder Migrationshintergrund.

Im Folgenden werden FörMig und BiSS bezüglich ihres Implementationsverständnisses verglichen. Neben der Fokussierung auf die Bildungssprache im Kontext einer durchgängigen Sprachbildung und der Bedeutung von Fortbildung und Professionalisierung verbindet die Programme die Vorstellung, dass sprachliche Bildung von Implementation als Prozess abhängig ist. Deutlich wird dies schon in der Anlage der Programme, die beide nicht vorrangig auf der Ebene von einzelnen Institutionen wie Kitas und Schulen agieren, sondern auf der Ebene von netzwerkartigen Zusammenschlüssen von Bildungseinrichtungen: „Basiseinheiten“ in FörMig bzw. „Verbünde“ in BiSS. Diese hatten den Auftrag, nicht einfach beschlossene Maßnahmen umzusetzen, sondern über die Zusammenarbeit eine durchgängige sprachliche Bildung in die Entwicklung ihrer Institutionen zu integrieren. 
Zwischen den beiden Programmen besteht aber ein Unterschied in der Konstruktion von Implementation. Ging es in FörMig vor allem darum, die vielfältigen Ansätze und Maßnahmen in den Bundesländern als heterogene Ausgangslage zusammenzubringen und nach dem Prinzip der Emergenz die Entstehung wirksamer Elemente zu beobachten (Gogolin et al. 2011, S. 20 ff.), um diese dann zu transferieren, so zielte BiSS von vornherein auf die Implementation von Elementen, deren Wirkung über wissenschaftliche Evaluationen nachgewiesen worden waren. In FörMig sollte also die Art und Weise der Implementation der bottom-up in das Programm eingebrachten Elemente beobachtet werden, um so Wirksames von weniger Wirksamem zu unterscheiden. In BiSS wiederum war Wirksamkeit vorausgesetzt und es stand - ganz im Sinne einer ,engeren“ Implementationsforschung - im Vordergrund, inwieweit die für eine erfolgreiche Wirkung notwendigen Bedingungen wie etwa die Konzepttreue angesichts von Adaptionen vor Ort erhalten blieben.

Aus der Anlage der Programme lassen sich Rückschlüsse auf die Strukturierung von Implementation als Prozess ziehen: FörMig war in zwei Phasen unterteilt, was auch die Anlage der wissenschaftlichen Evaluation steuerte: eine Implementationsund eine Vertiefungsphase (Gogolin et al. 2011, S. 115). Das resultierte aus der Annahme, dass die Implementationsphase mit einer Reihe von Unwägbarkeiten behaftet ist, die eine erfolgreiche Umsetzung zwar nicht vollständig verhindern, aber in unterschiedlichen Weisen behindern können. Dementsprechend war die Evaluation als Zwei-Kohorten-Design angelegt.

Verallgemeinert lässt sich sagen: Ein Implementationsprozess beginnt mit einer auch wilden Phase des Erprobens; es folgt eine disziplinierte Phase eher getreuen Einsatzes. Auf diese Weise kommt man über die beiden Programme zu einem idealtypischen Prozessmodell von Implementation, das eine Vorbereitungsphase, eine Umsetzungsphase und eine Überarbeitungsphase umfasst, bevor mit einer vollständigen Wirksamkeit zu rechnen ist. Ein Modell dafür liegt bei Fixsen et al. (2005) vor, welcher ausgehend von einer Suchphase mit dem Abschluss einer Entscheidung für ein zu implementierendes Konzept, seiner Installation, seiner Implementation und seiner dann vollen Funktionalität unterscheidet, was abschließend in die Innovation und Verstetigung übergeht, also den Transfer.

Das Verständnis von Implementation in BiSS ist in einem Beitrag von BeckerMrotzek und Butterworth (2018) skizziert. Dabei diente die BiSS-Expertise (Schneider et al. 2012) und die darin enthaltene Modulstruktur, die aus einer umfangreichen Sichtung vorliegender empirischer Studien resultierte, als „Orientierungsrahmen“ (Becker-Mrotzek und Butterworth 2018, S. 49) für die Steuerung. Nach Teerling et al. (2019, S. 7) handelt es sich um eine wissenschaftlich getriebene Top-downStruktur. Neben der Wirksamkeit geht es um eine notwendige „Adaptionsvarianz“ (Becker-Mrotzek und Butterworth 2018, S. 58). Gemeint ist damit eine Flexibilität von Konzepten, die als wesentliche Elemente die Leitung und eine Steuerungsgruppe, die Qualifizierung des Personals und seine Identifikation mit dem Konzept, die Akzeptanz sowie Verhaltensmuster der beteiligten Akteur*innen (Becker-Mrotzek und Butterworth 2018, S. 54) berücksichtigt. Wie Gräsel und Parchmann (2004) sowie Hasselhorn et al. (2014) empfehlen Becker-Mrotzek und Butterworth eine gestufte Vorgehensweise, welche die Einrichtung einer Steuerungsgruppe vorsieht, die das Feld vorbereitet und zunächst punktuelle Umsetzungen in „Innovationsinseln“ 
(Becker-Mrotzek und Butterworth 2018, S. 56) durchführt, bevor das Vorhaben auf einen breiteren Kreis von Akteur*innen ausgeweitet wird. Im Kontext sprachlicher Bildung ist das im Ausdruck ,durchgängige Sprachbildung“ formuliert: Über die horizontale Vernetzung sprachbildender Maßnahmen - sei es innerhalb einer Institution oder mit externen Partner*innen - kommen eine Reihe von unterschiedlichen Akteur*innen auf das Spielfeld, die gar nicht in direkter Linie der Steuerung stehen. Schon das für BiSS und die meisten anderen Programme charakteristische Setting von Bildungspolitik, Bildungsadministration, Bildungspraxis und Wissenschaft lässt erkennen, dass man nicht von linearen Steuerungsmodellen ausgehen kann, sondern die seitwärts gerichteten Krebsgänge sinnvollerweise einbezieht. So gesehen ist Adaptivität - und damit die Partizipation der Beteiligten - grundsätzlich eine Bedingung von Implementation.

\subsection{Ergebnisse aus der FörMig-Evaluation}

Es sollen an dieser Stelle auch Erkenntnisse aus den beiden Programmen gewürdigt und nicht nur strukturtheoretische Überlegungen zum Phänomen Implementation abgeleitet werden. Für FörMig lässt sich anführen, dass in diesem Programm der Blick auf Implementationsbedingungen breit entfaltet war; dementsprechend liegen auch eine Reihe von Ergebnissen vor. Davon ist einiges über die Programmevaluation methodisch abgesichert nachgewiesen, anderes ist aus der reichhaltigen Erfahrung der Programmbeteiligten verdichtetes Prozesswissen - auf den Ebenen der Praxis, der Bildungsadministration und der Wissenschaft.

In FörMig werden als Gelingensbedingungen resümiert: eine ,gemeinsame Sprache" zu finden, Kooperationsformate zu installieren, (sanktionsfreie) Zielvereinbarungen als Instrument der Verbindlichkeit einzugehen und Qualifizierungsmöglichkeiten für das pädagogische Personal anzubieten (Gogolin et al. 2011, S. 26). Als hilfreich für die Kooperation hat sich an den Übergängen die gemeinsame Diagnostik erwiesen (Gogolin et al. 2011, S. 32). Im weiteren fokussiert der Bericht folgende Bedingungen für eine gelingende Kooperation an den Schnittstellen: ausreichende zeitliche Ressourcen, Engagement von Einzelpersonen, verbunden mit Teambildung und Unterstützung durch die Leitungen, klare und transparente Organisation, realistische Zielsetzungen, Einbindung in die Schulprogrammarbeit und eine systematische Institutionsentwicklung (Gogolin et al. 2011, S. 34 ff.).

Hinsichtlich der Organisation schulischer Sprachbildung hat sich die unterrichtsintegrierte Förderung im allgemeinbildenden Schulsystem als effektiver herausgestellt als eine additive Förderung mittels Außendifferenzierung. Allerdings bezieht sich das lediglich auf den Unterricht in regulären Klassen mit ausdifferenzierten Fördermaßnahmen. Im Gegensatz dazu erwies sich die Förderung in Deutsch als Zweitsprache in speziellen Vollzeitklassen als überaus wirksam (Gogolin et al. 2011, S. 139). Eine Differenzierung der Wirkungen hinsichtlich inhaltlicher Merkmale der durchgeführten Förderungen (zum Lesen, zum Schreiben, zu textuellen Fertigkeiten) zeigte statistisch keine Effekte (Gogolin et al. 2011, S. 136f.); lediglich die Konzentration auf elementare Sprachfertigkeiten im Übergang in die Sekundarstufe (Gogolin et al. 2011, S. 139). Gruppengröße und sprachliche Homogenität in den 
Fördergruppen hatten keinen nachweisbaren Einfluss auf die Ergebnisse (Gogolin et al. 2011, S. 138).

Neben dieser Auswertung der Daten im Hinblick auf die Organisation der Förderung wurde in der Evaluation ein zweiter Schritt getan, es wurden nämlich erfolgreiche Lerngruppen und Basiseinheiten identifiziert, um herauszufinden, was sie erfolgreich werden ließ. Dazu wurden die Testergebnisse auf die konkrete Durchführung der sprachlichen Bildungsarbeit in den Basiseinheiten hin modelliert. Dabei traten einige Erfolg induzierenden Elemente in den Blick: Netzwerkarbeit, gemeinsame Konzeptentwicklung, Entwicklung und Erprobung von Fördermaterialien, intensive institutionenübergreifende Kooperation (auch mit Hospitationen), Systematisierung der Sprachförderung, gemeinsame Vereinbarungen zu Förderstrategien und Arbeitsfeldern, Fortbildungen (Gogolin et al. 2011, S. 150 ff.). ,Erfolgreiche Fördergruppen agieren nicht isoliert", fassen die Autor*innen ihre Ergebnisse zusammen; dazu gehören die Verbindlichkeit des gemeinsamen Vorgehens, regelmäßiger Austausch und Feedback sowie die Verankerung der Förderung im regulären Unterricht (Gogolin et al. 2011, S. 164f.). Die Autor*innen weisen außerdem auf einen Befund hin, der das Thema Konzepttreue als Implementationsbedingung betrifft: Danach beeinflusst nicht nur der Grad der praktischen Realisierung die Wirksamkeit eingesetzter Maßnahmen, sondern auch deren Qualität (Gogolin et al. 2011, S. 165). So kann es auch sein, dass eine Adaption, die zwar deutlich vom ursprünglichen Konzept abweicht, aber qualitativ hochwertig durchgeführt wurde, zu positiven Ergebnissen führt.

\subsection{Ergebnisse aus den BiSS-Evaluationsprojekten ${ }^{3}$}

In BiSS war das Trägerkonsortium für Beratung und Unterstützung der Evaluation der Verbünde zuständig; die Evaluationsvorhaben selbst wurden von externen Forschungsteams durchgeführt. Die Ergebnisse liegen noch nicht vollständig vor. In Anbetracht dieser Situation werden im Folgenden keinesfalls alle Erkenntnisse zur Implementation als Gestaltungsfeld im Detail einbezogen, sondern lediglich die Haupttendenzen zusammengefasst und den Erfolgskriterien nach Souvignier und Philipp (2016) zugeordnet, die hier um das Kriterium Kompetenz erweitert werden. Es handelt sich um Faktoren, die

- in der Person der Lehrkräfte bzw. pädagogischen Fachkräfte liegen (Akzeptanz, Kompetenz),

- als Bedingungen eines wirksamen Unterrichts bzw. der pädagogischen Arbeit gelten können (Angemessenheit, Wiedergabetreue, Durchdringung),

- die Rahmenbedingungen auf Ebene der Institutionen wie auch der BiSS-Verbünde darstellen (Machbarkeit),

- auf der übergeordneten Entscheidungsebene der Steuerung angesiedelt sind, i.d. R. der Bildungsadministration (Implementationskosten, Nachhaltigkeit),

\footnotetext{
3 Die Ergebnisse liegen derzeit in Form eines Projektatlasses (Henschel et al. 2014), einem Sammelband (vgl. Titz et al. 2018) sowie einer Reihe verschiedener Veröffentlichung der beteiligten Wissenschaftler*innen vor. Ein zusammenfassender Band in der BiSS-Reihe ist im Erscheinen. Ein weiterer Projektatlas mit den Ergebnissen aus 37 Begleitprojekten zu BiSS-Projekten wird in zwei Tranchen 2020/21 erscheinen.
} 


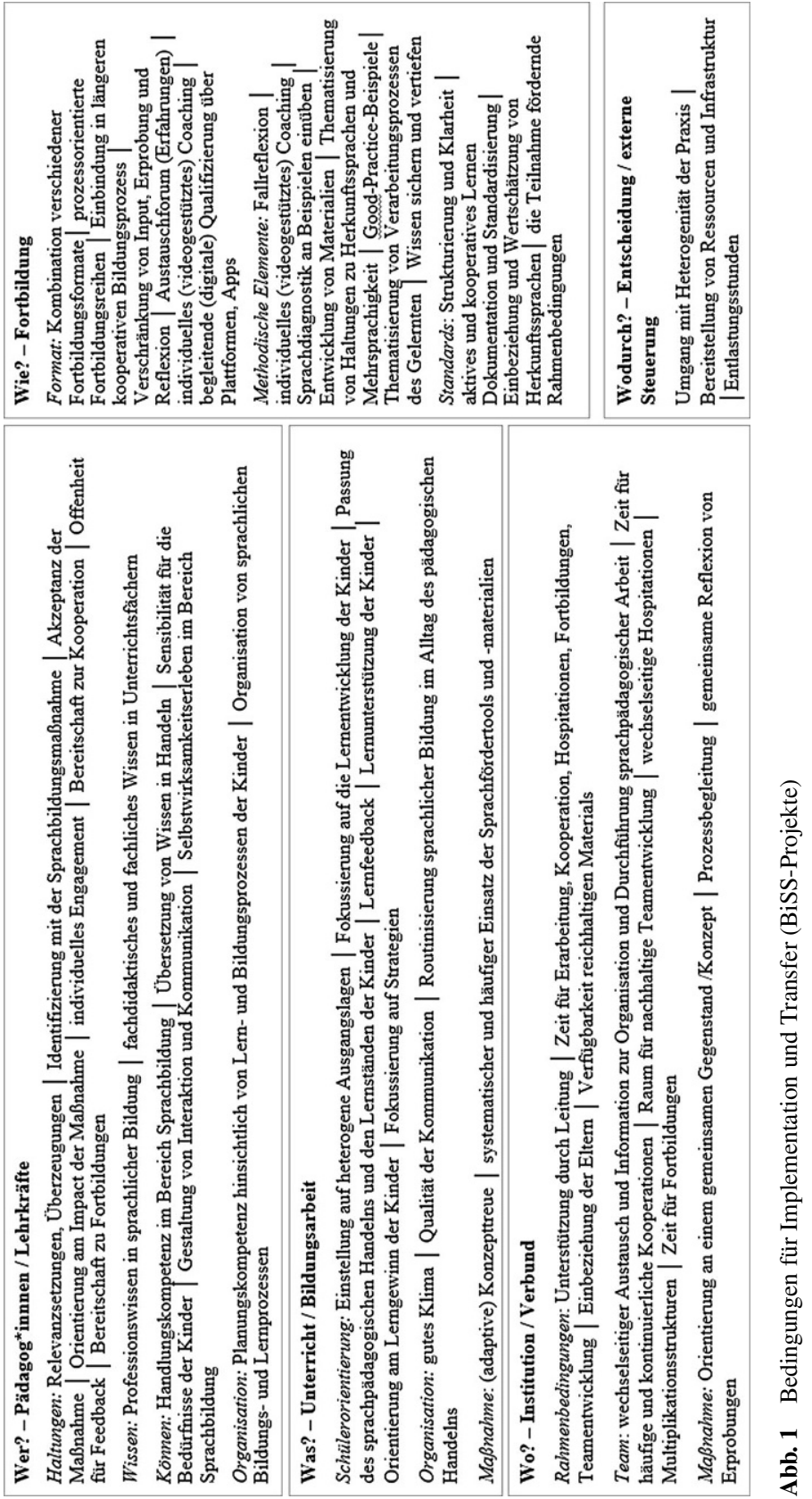


- im Rahmen von Fortbildungen zur Professionalisierung der Pädagog*innen dienen (Kompetenz).

Die Ebene der Kinder bzw. Schüler*innen wird - abgesehen vom allgemeinen Ausgangspunkt einer großen Heterogenität der Lernausgangslagen - selbst nicht thematisiert, bildet als abhängige Variable allerdings die Zielebene all dessen, worauf die Maßnahmen der sprachlichen Bildungsarbeit letztlich ausgerichtet sind. Die Befunde reihen sich einerseits in die Beobachtung bei Schrader et al. (2020, S. 29) ein, dass Implementationsstudien überwiegend auf der Mesoebene angesiedelt sind; andererseits kommt der Mikroebene sowie der Ebene der Professionalisierung und Qualifizierung ein großes Gewicht zu. Hingegen ist die bildungspolitische Makroebene als wichtiger Kontext nicht enthalten; Hinweise auf die externe Steuerungsebene (Wodurch?) lassen sich nur wenige finden. Dies ist der Konstruktion von groß angelegten Programmen geschuldet, deren Einsetzung stets ein umfangreicher politischer Prozess der Entscheidungsfindung vorausgeht. Angesichts des Umfangs und der Menge der Studien stellen wir diese nicht einzeln vor, sondern fassen sie tabellarisch zusammen (vgl. Abb. 1); die dazu ausgewerteten Studien befinden sich in der Übersicht im Anhang (vgl. Tab. 1).

Die Wirkfaktoren lassen sich auf der Ebene der Akteur*innen (Wer?) recht gut mittels eines geläufigen Kompetenzmodells, sprich in Einstellungen und Haltungen sowie Wissen und Können abbilden. Hinzu tritt eine spezifische Kompetenzebene, die sich als die Organisation von sprachlichen Bildungsprozessen in der Praxis beschreiben lässt und eine Planungskompetenz hinsichtlich der Lern- und Bildungsprozesse der Kinder einschließt. Auf der Ebene des Unterrichts bzw. der sprachlichen Bildungsarbeit (Was?) lassen sich drei Dimensionen unterscheiden: die Ausrichtung auf die Schüler*innen und ihre spezifischen Lernbedingungen und der entsprechenden Gestaltung der Lern- und Bildungsräume sowie spezifische Orientierungen hinsichtlich der Umsetzung und des Einsatzes einer gewählten Maßnahme (Konzepttreue). Die Ebene der Institution bzw. des Verbunds (Wo?) enthält die Dimensionen der Rahmenbedingungen und einen starken Fokus auf Fragen der Kooperation und Teamentwicklung. Auch hier lässt sich wieder eine Konzentration auf die gemeinsame Maßnahme, ihre Begleitung im Prozess und Reflexion erkennen. Sprachliche Bildungsarbeit ist demnach ganz eindeutig kein singuläres Geschäft. Ebenso sind ihre Implementation und ihr Transfer nichts einfach Vorhandenes, sondern bedürfen spezifischer Qualifizierungs- und Professionalisierungsmaßnahmen. Eine vierte Ebene fasst dementsprechend die Faktoren für Fortbildungen (Wie?) zu wirksamen Sprachbildungsmaßnahmen zusammen. Dabei lässt sich eine Differenzierung der vorliegenden Ergebnisse hinsichtlich der Formate von Fortbildungen sowie der methodischen Elementen erkennen.

\subsection{Maßnahmen sprachlicher Bildung im Elementarbereich in Deutschland}

Mit einer Übersicht über Interventionsstudien zu Sprachfördermaßnahmen zeigen Egert et al. (2020, S. 15 f.), dass wirkungsvolle Sprachförderung einer guten Umsetzungsqualität bedarf, wofür eine ausreichende Qualifizierung und Unterstützung der pädagogischen Fachkräfte als notwendig erachtet wird. Zur Erhöhung der Effekti- 
vität von Sprachfördermaßnahmen stellen Fortbildungen von pädagogischen Fachkräften im Elementarbereich also eine wichtige Maßnahme dar. Auch hier sind es vornehmlich Implementationsstudien im Rahmen von Evaluationen und Interventionen mit einem Kontrollgruppendesign, die der Frage nachgehen, inwiefern sich die Sprachförderkompetenz der Fachkräfte durch gezielte Weiterbildungsmaßnahmen erhöhen. Dem liegt die Annahme zugrunde, dass sich eine erhöhte Sprachförderkompetenz der pädagogischen Fachkräfte auf die sprachlichen Fähigkeiten der Kinder auswirkt. Diese Implementationsstudien seien im Folgenden kurz im Einzelnen dargestellt, um die Zusammenhänge von Wirkungen auf den Ebenen der Kinder und der pädagogischen Fachkräfte mit den Bedingungen der Implementation zu zeigen; eine weitere Anreicherung der in Abb. 1 zusammengefassten Wirkfaktoren ergibt sich daraus nicht.

Kammermeyer et al. (2019) untersuchten in einer Prä-Post-Follow-up-Studie zwei Weiterbildungsansätze im Feld. In der Interventionsgruppe wurde die Weiterbildung „Mit Kindern im Gespräch“ (Kammermeyer et al. 2017) durchgeführt, während die Kontrollgruppe an der regulären und inhaltlich breiten Sprachfördermaßnahme des Landes Rheinland-Pfalz „Sprache - Schlüssel zur Welt“ teilnahm. Anhand von standardisierten Videobeobachtungen wurde die Häufigkeit der Nutzung komplexer Sprachförderstrategien von pädagogischen Fachkräften erfasst. Die Ergebnisse zeigen, dass die pädagogischen Fachkräfte der Interventionsgruppe signifikant häufiger komplexe Modellierungs- und Fragestrategien und zudem signifikant häufiger komplexe Strategien zur Konzeptentwicklung einsetzten als die Vergleichsgruppe; und zwar sowohl direkt nach der Weiterbildung als auch ein Jahr danach (Kammermeyer et al. 2017, S. 219). Mit dem Follow-Up-Test wurde somit das Implementationskriterium der Nachhaltigkeit berücksichtigt. Die Autorinnen erwarten, dass sich aufgrund der erhöhten sprachförderlichen Aktivität auch die sprachlichen Kompetenzen der Kinder verbessern werden; allerdings wurden diese im Rahmen der Studie nicht erfasst.

Lemmer et al. (2019) untersuchen die Effektivität einer linguistisch fundierten Sprachförderung mit einem Prä-Posttest-Kontrollgruppen-Design sowohl bei den pädagogischen Fachkräften als auch bei Kindern. Hierfür wurden zum einen die Sprachförderkompetenz der pädagogischen Fachkräfte mit dem Testverfahren SprachKopf und zum anderen die Sprachfähigkeiten mehrsprachiger Kinder mit dem Testverfahren LiSe-DaZ gemessen. Die pädagogischen Fachkräfte erhielten eine Fortbildungsmaßnahme und wurden im Anschluss mit einer Wartekontrollgruppe verglichen. Die Autor*innen belegen zum einen, dass die Sprachförderkompetenz der Interventionsgruppe anders als in der Vergleichsgruppe im Bereich Syntax signifikant anstieg, nicht hingegen in der Kasusmorphologie (Lemmer et al. 2019). Die Autor*innen sehen insgesamt die Wirksamkeit der linguistisch fundierten Weiterbildung auf der Ebene der pädagogischen Fachkräfte bestätigt, allerdings wurde nicht untersucht, ,welche [pädagogische Fachkraft] innerhalb der Einrichtungen regelmäßig förderten und inwieweit die Sprachförderkonzepte fachgerecht in der Praxis umgesetzt wurden“ (Lemmer et al. 2019, S. 185), sodass u. a. die Implementationskriterien der Nachhaltigkeit und Konzepttreue offen bleiben.

Die Studie von Simon und Sachse (2013) untersucht die Wirksamkeit des „Heidelberger Interaktionstrainings für pädagogisches Fachpersonal zur Förderung ein- 
und mehrsprachiger Kinder" (HIT). Mit einer Prä-Post-Follow-up-Studie wurde die Sprachförderkompetenz der pädagogischen Fachkräfte erhoben, darüber hinaus die Sprechfreude der Kinder, indem die Summe kindlicher Äußerungen und ihr Redeanteil an der gesamten Kommunikation in Videointeraktionsanalysen berechnet wurde (Lemmer et al. 2019, S. 385). Darüber hinaus wurden mithilfe von Testbatterien sprachliche Teilbereiche, wie z.B. Wortschatz und das phonologische Arbeitsgedächtnis, untersucht (Lemmer et al. 2019, S. 386). Nachgewiesen wurden Interventionseffekte auf die pädagogischen Fachkräfte (Simon und Sachse 2011). Die Analysen zeigen außerdem, dass signifikante Effekte in Hinblick auf Sprechfreude, Kommunikationsanteile sowie längere sprachliche Äußerungen bei den Kindern bestehen, die von den pädagogischen Fachkräften in der Interventionsgruppe gefördert wurden. Auf die im Rahmen der Testbatterien erhobenen Bereiche hingegen ließen sich keine globalen Effekte nachweisen, sondern nur bei den Kindern mit besonders niedrigen Anfangskompetenzen (Simon und Sachse 2013, S. 393). Die Autor*innen bewerten das HIT aufgrund der kurzen Fortbildungsdauer als eine besonders ökonomische Fortbildung und rücken damit neben der Effektivität auf Ebene der pädagogischen Fachkräfte und ihrer Qualifizierung auch das Implementationskriterium der Machbarkeit in den Vordergrund.

Sprachfördermaßnahmen mit einer heterogenen Umsetzung, geringer Konzepttreue sowie geringer „Förderdosis“ zeigen hingegen keine Wirksamkeit; das erbringen die Studien von Sachse et al. (2012) zum Programm „Deutsch für den Schulstart“ sowie von Groth et al. (2017) zum Sprachförderprogramm KIKUS (vgl. auch Egert et al. 2020, S. 16f.). Die spracherwerbstheoretischen Grundlagen der Programme wurden allerdings nicht überprüft, sodass offenbleibt, ob die fehlende Wirksamkeit auf die Implementationsebenen der Umsetzung und der Machbarkeit zurückzuführen ist oder auf die Programme an sich (Egert et al. 2020, S. 17). Sachse et al. (2012, S. 199) diskutieren die fehlende Wirksamkeit allerdings mit Blick auf das Implementationskriterium der Umsetzungstreue, und zwar dahingehend, dass die Umsetzung der Sprachförderung variierte und Kinder zu großen Teilen nicht altersangemessene Fördereinheiten erhielten.

Deutlich wird im Kontext der Elementarbildung eine Fokussierung der Studien auf die Wirksamkeit von Sprachfördermaßnahmen im Zusammenhang mit der Qualifizierung der pädagogischen Fachkräfte, sodass an dieser Stelle eine Verzahnung von Implementations- und Evaluationsforschung festgehalten werden kann. Umfängliche Implementationsstudien im ,engeren Sinne“ stehen auch hier aus. Die berücksichtigten Implementationsebenen beziehen sich auf pragmatische Aspekte, d.h. die Machbarkeit, die Dauer der Fortbildung, sowie die Wirksamkeit der Programme und ihre Nachhaltigkeit im Rahmen von Follow-up-Erhebungen. Offen bleiben vor allem die Ebenen der Akzeptanz sowie auch die Perspektiven der Akteur*innen. Die Bedeutung der Kompetenzerweiterung durch Qualifizierung ist klar belegt.

Wie gesagt konzentrieren sich die bundesweiten Programme letztlich auf die sprachliche Bildung im Deutschen. Das erfolgt durchaus in unterschiedlicher Intensität. Aber wie steht es mit der Mehrsprachigkeit als Aktivposten? Das soll im Folgenden am Beispiel von Studien zur Implementation des Translanguaging in verschiedenen Bildungskontexten betrachtet werden. 


\section{Zur Berücksichtigung der Mehrsprachigkeit in der sprachlichen Bildung - Translanguaging als Beispiel}

Mit dem Ausdruck Translanguaging wird linguistisch der Sprachgebrauch mehrsprachiger Sprecher*innen bezeichnet; demnach haben diese aus der eigenen Innenperspektive heraus ein Gesamtrepertoire an sprachlichen Mitteln, dessen sie sich kontextsensibel bedienen können. Die Abgrenzbarkeit von Einzelsprachen wird somit aus der Perspektive der Sprechenden in Frage gestellt. In diesem Sinne wird das Konzept derzeit zu einer Theorie der angewandten Linguistik weiterentwickelt (Otheguy et al. 2015; Wei 2018). Im Folgenden steht Translanguaging als sprachpädagogisches Konzept im Umgang mit Mehrsprachigkeit im Unterricht bzw. dem pädagogischen Alltag im Vordergrund. Dieses geht auf die Arbeiten des Lehrers Cen Williams zurück. Dieser führte im Jahr 1994 den walisischen Begriff trawsieithu ein, um eine Methode zu beschreiben, die im Rahmen von Revitalisierungsmaßnahmen für die walisische Sprache sowohl diese als auch die englische Sprache systematisch in den Unterricht einbezog (vgl. García und Lin 2016; Wei 2018). Das Konzept stützt sich normativ auf einen wertschätzenden Umgang mit Mehrsprachigkeit und mehrsprachigen Sprecher*innen und vollzieht organisatorisch einen Bruch mit der Idee der strikten Sprachtrennung im Unterricht (Lewis et al. 2012, S. 643). Der Fokus liegt damit nicht mehr auf den Sprachen mit ihren Teilsystemen. Stattdessen wird das sprachliche Handeln mehrsprachiger Sprecher*innen und ihr gesamtes sprachliches Repertoire in den Mittelpunkt gerückt.

Translanguaging ist somit kein neues Phänomen, sondern kann als eine neue Perspektive auf bestehende sprachliche Praktiken mehrsprachiger Sprecher*innen verstanden werden. Es trägt zur Überwindung einer Sichtweise der strikten Sprachentrennung bei. Es ist darauf hinzuweisen, dass solch eine Perspektive in Studien eingenommen wurde, ohne dies unter dem Konzept des Translanguaging zu fassen. Das gilt z.B. für Stevenson (2013), der rekonstruiert, wie englisch-spanischsprachige Schüler*innen in den USA ihr sprachliches Repertoire im naturwissenschaftlichen Unterricht einsetzen. Für Deutschland zeigen Schüler-Meyer et al. (2018), dass die Einbeziehung aller sprachlichen Kompetenzen von Schüler*innen im Mathematikunterricht sich bei Lernenden, die Kompetenzen in der deutschen und in der türkischen Sprache besitzen, positiv auf den Lernzuwachs in der Bruchrechnung auswirkt. Aktuelle erziehungswissenschaftliche Fragen beschäftigen sich damit, wie eine Didaktik des Translanguaging weiterentwickelt werden kann (so z. B. García und Lin 2016), nachdem das Konzept seit einigen Jahren international eine größere Verbreitung gefunden hat. Einen aktuellen Überblick zu theoretischen Grundlagen und zur didaktischen Entwicklung des Konzepts bieten García und Kleifgen (2020).

Wie sieht es mit der Rezeption in Deutschland aus? Hier wird Translanguaging erst seit einigen Jahren als pädagogisches Konzept diskutiert. Aktuelle Arbeiten beschäftigen sich mit konzeptionellen Fragen sowie damit, wie Translanguaging im pädagogischen Alltag der Frühpädagogik sowie an Schulen im Sinne einer inklusiven Bildung implementiert werden kann (Panagiotopoulou 2016, 2018). Für die Schule erachten Fürstenau et al. (2020) das Konzept des Translanguaging als eines, welches den Lehrkräften die Möglichkeit eröffnet, die Ressourcen der Schüler*innen einzubeziehen sowie mit sprachlichen Unsicherheiten umzugehen, ohne 
selbst die Sprachen beherrschen zu müssen. Translanguaging schließt somit auch an bereits bestehende und bewährte Konzepte an - so z. B. dem Ansatz der Language Awareness. Den gedanklichen Boden haben dem die Arbeiten von Gogolin bereitet, die bereits 1984 ein Konzept von Gesamtsprachigkeit zur Umsetzung vorschlug und 1997, transformationelle sprachliche Kompetenzen als Zieldimension nicht nur sprachdidaktisch entwarf, sondern auch bildungstheoretisch verankerte (Gogolin 1997, S. 344).

Im vorliegenden Beitrag werden jene Studien aufgegriffen, die sich explizit auf das Konzept des Translanguaging und seiner Verbreitung in Bildungsinstitutionen beziehen. Bislang liegen (noch) nicht sehr viele Studien dazu vor. Das scheint sich aber gerade zu ändern, denn immerhin ist Translanguaging das Konzept, zu dem sich in jüngerer Zeit überhaupt eine nennenswerte Anzahl an Studien zu Fragen von Implementation und Transfer recherchieren ließ. Hinsichtlich der Zielgruppen sind diese breit gestreut und erstrecken sich über die gesamte Bildungslaufbahn vom Elementarbereich über die Schule bis hin zur Universität. Neben der Heterogenität hinsichtlich der Altersgruppen besteht eine weitere darin, dass sich einige der Studien nicht auf die sprachliche Bildung lebensweltlich mehrsprachiger Kinder und Jugendlicher richten, sondern Translanguaging für den Fremdsprachenunterricht nutzen. Für die betrachteten Untersuchungen lassen sich zwei thematische Schwerpunkte erkennen: Zum einen wird mittels des Einsatzes von Video- oder Audioaufnahmen sowie ethnografischen Beobachtungen untersucht, wodurch sich translinguale Praktiken von Kindern und Schüler*innen auszeichnen. Zum anderen wird der bildungspraktische Einsatz im Hinblick auf die Entwicklung einer Didaktik des Translanguaging betrachtet.

Auf der forschungsmethodischen Ebene lässt sich eine Dominanz von Fallstudien beobachten, insbesondere von Studien, die eine Zielsetzung in der didaktischen Forschung haben und berichten, wie Translanguaging eingesetzt und als sprachpädagogisches Konzept weiterentwickelt werden kann (vgl. dazu auch García und Kleifgen 2020, S. 561). Die Studien erfassen fast ausschließlich kleine Samples, in der Regel eine oder mehrere Lerngruppen. Zwar gibt es inzwischen auch zumindest eine Studie, die mit testförmigen Instrumenten und Vergleichsgruppen arbeitet (Gantefort und Maahs 2020); allerdings liegen dazu bislang die konkreten Ergebnisse noch nicht vor. Wirkungsstudien sind selten. Als einzige untersuchte Beres (2015) in einer experimentellen neurowissenschaftlichen Studie mittels EEG neben der Messung der Abrufgeschwindigkeit auch Event-Related Potentials (ERP) zur Erfassung des Effekts des Translanguaging beim Wissenserwerb (auf der Ebene von Einzelwörtern) bei 34 Erwachsenen in Wales. Sie fand einen eindeutigen Effekt für den Vorteil des Translanguaging, indem sie die Ergebnisse mit Kontrollitems verglich, die in einem monolingualen Kontext erhoben worden waren. Die semantische Verarbeitung erfolgte nach den ERP-Ergebnissen deutlich schneller, auch wenn die Messung der Abrufzeiten keine Unterschiede ergab und die Proband*innen die zweisprachige Testsituation subjektiv als anstrengender erlebten. In Follow-Up-Messungen zeigten sich diese Vorteile stabil, auch nach zwei und vier Wochen. Die Studie gibt zwar keine Auskunft über Implementationsbedingungen in Schule und Erwachsenenbildung, erbringt aber den Nachweis der prinzipiellen Wirksamkeit auf Lernprozesse. 
Auffällig ist auch, dass eine Reihe der Studien zu Translanguaging in Unterrichtssettings außerhalb des regulären Unterrichts in Projekten, häufiger auch unter Beteiligung von Hochschulen durchgeführt wurden; das gilt für internationale wie auch in Deutschland durchgeführte Studien. Darüber wird zum einen erkennbar, dass das Thema noch nicht im Zentrum einer didaktischen Selbstverständlichkeit sprachlicher Bildung angekommen ist, sondern sich in einer Phase des Ausprobierens und Experimentierens befindet. Zum anderen markiert es die Bedeutung der sog. symbiotischen Implementationen mittels enger Kooperationen zwischen Praktiker*innen und Wissenschaftler*innen hinsichtlich der Entwicklung und des Einsatzes von Innovationen im Bereich sprachlicher Bildung.

Eine Studie von Duarte (2019) gibt Hinweise auf mögliche Gründe für die noch geringe Verbreitung des Translanguaging in Deutschland. Sie untersuchte Videoaufnahmen aus dem Unterricht von immerhin 59 zehnten Klassen aus vier weiterführenden Schulen mit großen Zahlen von mehrsprachigen Schüler*innen in Deutschland und analysierte das Vorkommen translingualer Interaktionspraxen mit der Methode der soziokulturellen Diskursanalyse nach Mercer (2004). Diese nahmen lediglich einen Umfang von gerade fünf Prozent ein und erfolgen zu $90 \%$ unterrichtsbezogen zur Lösung von Aufgaben. In den meisten Fällen handelte es sich um einen Typus des Sprechens, der als ,exploratory talk“ charakterisiert wird, d. h. behauptende und darstellende Sprachhandlungen, gefolgt von Fragen, Informationen, Bestätigungen, Paraphrasen und Zitationen sowie Argumentationen und dem Verhandeln von Meinungen und Wortbedeutungen. In den Beispielen wurden die Schüler*innen im Unterricht nie explizit dazu aufgefordert, ihr gesamtes linguistische Repertoire zu nutzen. Im Ergebnis sieht die Autorin die Dominanz des Deutschen als Unterrichtssprache bestätigt; ein mehrsprachiges Unterrichten mittels Translanguaging ist also nach wie vor ein Desiderat.

Die für Fragen von Implementation und Transfer relevanten Ergebnisse aus den Studien werden wie zuvor in einer Tabelle zusammengefasst (vgl. Abb. 2). Diese fällt deutlich übersichtlicher aus als die zuvor allein für den Kontext des BundLänder-Programms „Bildung durch Sprache und Schrift“ (BiSS) zusammengestellte Tabelle. Um jedoch den Eindruck zu vermeiden, dass es sich hierbei um ein zusammenhängendes Feld mit einem gemeinsamen Fokus von Studien handelt und um die unterschiedlichen Zugänge nachvollziehen zu lassen, werden einige von diesen im Folgenden kurz einzeln dargestellt. Die Gliederung erfolgt nach der bildungsbiographischen Reihenfolge, d.h., es beginnt mit dem Elementarbereich und endet in der Hochschulbildung.

Für den Elementarbereich geben einige Studien Hinweise auf zu beachtende Implementationsbedingungen. Ausgehend von einer Kritik an der Dominanz des Englischen in Bildungsinstitutionen beschreibt Pino (2019) in einer autoethnografischen Studie ihren Unterricht in einem Kindergarten, wo sie explizit translinguale Räume (Translanguaging Spaces) einrichtet: In diesen sind translinguale Praktiken nicht nur erlaubt, die Kinder werden explizit dazu ermutigt. Die Autorin kann festhalten, dass die Kinder translinguale Praktiken einsetzen, um zu signalisieren, dass sie etwas verstanden haben, ihr Wissen darzulegen und um den Konversationsfluss nicht zu unterbrechen. Außerdem konnte sie beobachten, dass es die Schüler*innen dazu befähigte, ihre eigenen Gedanken und Ideen zu verbalisieren und eine sprach- 
H.-J. Roth et al.
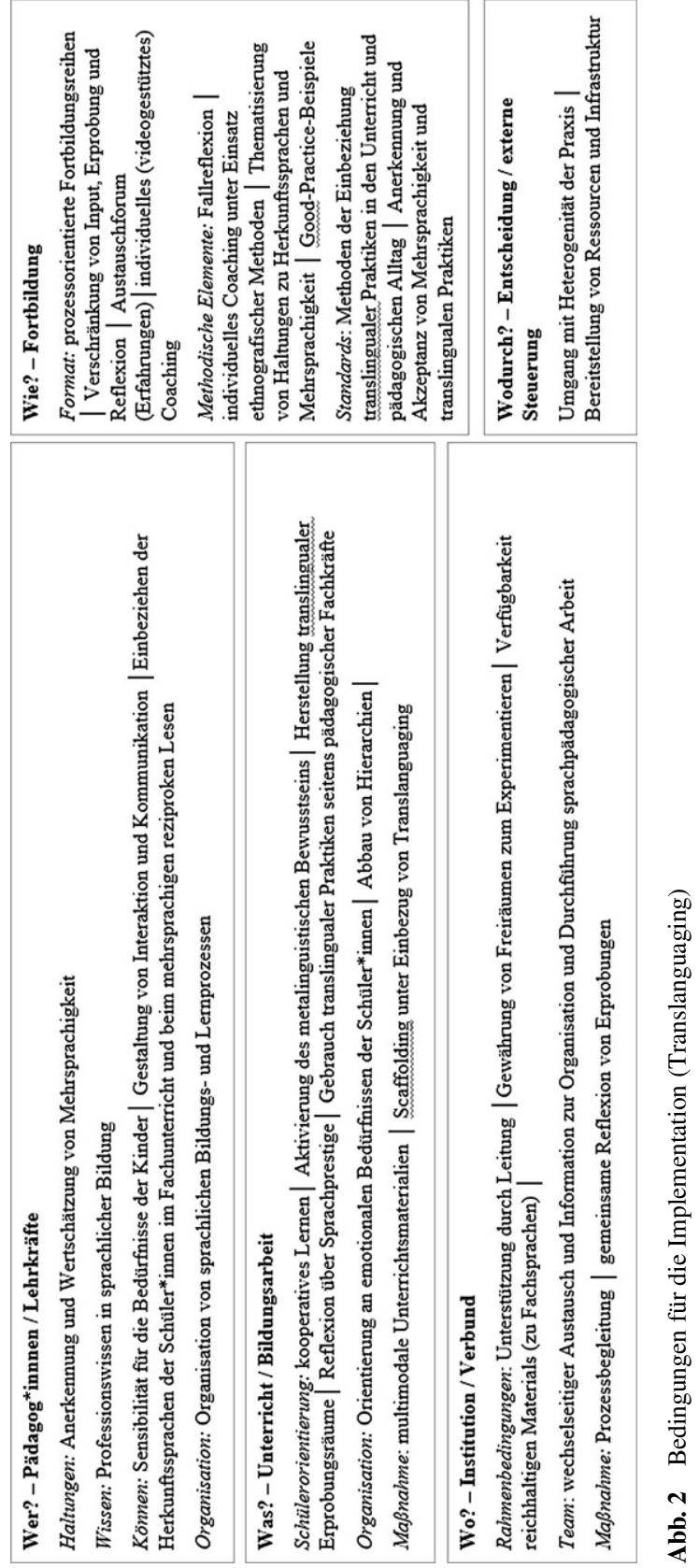
liche Identität auszubilden. Daraus leitet sie weitere Implikationen für die Praxis ab, wobei die Akzeptanz translingualer Praktiken unter pädagogischen Fachkräften im Vordergrund steht.

Kirsch und Seele (2020) zeigen mittels Beobachtungen verschiedene Funktionen translingualer Praktiken im Handeln der pädagogischen Fachkräfte, so z. B. Übersetzungen, und den Einsatz von Familiensprachen. Nach ihren Ergebnissen erhöht der Einsatz translingualer Praktiken auf Seiten der pädagogischen Fachkräfte deren Nutzung durch die Kinder; mehrsprachiges Agieren wird anscheinend zu einer legitimen Praktik im pädagogischen Alltag (Kirsch und Seele 2020, S. 77). Die Autorinnen weisen darauf hin, dass die Perspektiven der Kinder und der Eltern wie auch der politischen und institutionellen Kontexte bislang $\mathrm{zu}$ wenig erforscht sind (Kirsch und Seele 2020, S. 78).

Für die Studien zum Translanguaging im Elementarbereich zeigt sich also, dass diese stärker darauf abzielen, den Kindern einen wertschätzenden und anerkennenden Raum für ihre sprachlichen Praktiken zu schaffen. Diese Kompetenz über Fortbildungen zu vermitteln, ist die Zielsetzung in weiteren Studien: Ausgehend von Forschungsergebnissen, dass auch mehrsprachige Fachkräfte in der Frühpädagogik Skepsis gegenüber der Implementation von Mehrsprachigkeit in den pädagogischen Alltag aufweisen (vgl. z. B. Kratzmann et al. 2017), entwickeln Panagiotopoulou und Hammel (2020) ein Trainingskonzept für mehrsprachige pädagogische Fachkräfte und Lehrkräfte an Grundschulen mit dem Ziel, Translanguaging zu implementieren und so monolinguale Normvorstellungen im pädagogischen Alltag zu überwinden. Angehende Fach- und Lehrkräfte sollen in ihrem Alltag unter Einsatz ethnografischer Methoden begleitet werden, um anschließend gemeinsam das pädagogische Handeln zu reflektieren (Panagiotopoulou und Hammel 2020, S. 211).

Für die Schule lassen sich zudem eine Reihe weiterer Studien anführen, die darauf zielen, translinguale Praktiken von Schüler*innen in den Unterricht einzubeziehen, um diese für ihre Lernprozesse fruchtbar zu machen.

Das gilt z.B. für die Studie von Aghai et al. (2020) zum Unterricht in Englisch als Zweitsprache, die zum einen mittels Unterrichtsaufnahmen und Interviews mit zehn Lernenden eine Stärkung des metalinguistischen Bewusstseins durch Translanguaging bereits in einem frühen Stadium des Zweitspracherwerbs nahelegt und zum anderen für die Weiterentwicklung des didaktischen Konzepts Praktiken des Translanguaging herausarbeitet und Schlussfolgerungen für die didaktische Implementation des Konzepts zieht. Dabei betonen die Autor*innen, insbesondere die Notwendigkeit einer positiven Einstellung gegenüber mehrsprachigen Potenzialen, eine Aktivierung des metalinguistischen Bewusstseins und die Fort- und Ausbildung von Lehrkräften (Aghai et al. 2020). In einer ähnlichen, umfangreicher angelegten Studie mit ausführlichen Beobachtungen der Interaktionen in drei Lerngruppen im Unterricht eines Englischintensivprogramms an der Universität hat dieselbe Autorin (Aghai 2019) den Einfluss der Sprachideologien von drei Lehrkräften auf ihren Unterricht und die Nutzung des Translanguaging sowie ihre Schüler*innen untersucht. Im Ergebnis wirkt es sich deutlich aus, ob die Lehrkräfte Mehrsprachigkeit als Problem, als natürlichen Prozess oder als Ressource einordnen (z. B. dahingehend, wie sich die Lernenden Wissen aneignen), und ob Translanguaging als didaktisches Konzept gemieden, geduldet oder akzeptiert wird. Aghai weist auch darauf hin, 
dass eigene Mehrsprachigkeit von Lehrkräften nicht unmittelbar in eine Kompetenz translingualer Unterrichtsgestaltung einmünde, sondern einer spezifischen Ausbildung bedürfe.

Karlsson et al. (2020) legen mittels Video- und Audioaufnahmen von Unterrichtsstunden in einer schwedischen Grundschule dar, wie translinguale Praktiken im naturwissenschaftlichen Unterricht seitens der Schüler*innen eingesetzt werden und welche möglichen Hürden für das fachliche Lernen aufkommen. Die Autor*innen zeigen z. T. anhand von Fallbeispielen, wie Schüler*innen mittels Translanguaging auch bei geringen Schwedischkenntnissen in das fachliche Lernen einbezogen werden und wie sie durch die Einbeziehung ihres sprachlichen Repertoires gemeinsam Wissen erschließen können. Eine Grenze sehen sie für den Fachunterricht darin, dass die sprachlichen Kenntnisse der Schüler*innen nur alltagssprachlich verfügbar sind. Sie fordern daher eine Weiterentwicklung mit zusätzlichen unterstützenden (multimodalen) Unterrichtsmaterialien und Unterstützung auf der Ebene der Schulorganisation.

In einem deutschen Forschungsprojekt wird Translanguaging als systematischer Baustein im Rahmen von mehrsprachigen Grundschulklassen, die nach dem Prinzip der Koordinierten Alphabetisierung (KOALA) unterrichtet werden, untersucht (vgl. Gantefort und Maahs 2020; Gantefort und Sánchez Oroquieta 2015). Mit Bezug auf das Konzept des Translanguaging wurde in einem BISS-Verbund aus vier Grundschulen das „mehrsprachige reziproke Lesen“ entwickelt. Unter Einsatz dieser Methode können Lehrkräfte ihre Schüler*innen dabei unterstützen, sich mithilfe ihres gesamtsprachlichen Repertoires fachliches Wissen, Leseverstehen sowie metasprachliche Fähigkeiten anzueignen (Gantefort und Maahs 2020, S. 5). Für die fünf Schritte des Reziproken Lesens (Vorlesen, Wörter und Textstellen klären, Fragen stellen, Zusammenfassen und Vorhersagen) wird die Möglichkeit eröffnet, die den Schüler*innen zur Verfügung stehenden sprachlichen Mittel einzusetzen, indem Gruppen mit ähnlichen Sprachkenntnissen zusammengesetzt werden. Im Rahmen einer Begleitstudie ist eine Implementierung in acht Lerngruppen geplant. Der Fokus liegt u. a. auf den Effekten mehrsprachiger Interaktionen der Lernenden auf die Entwicklung von Leseverstehen im Deutschen und Türkischen. Es werden ebenso Effekte einer mehrsprachigen familiären Lernumwelt untersucht.

Hansen-Thomas et al. (2020) führten eine Fallstudie anhand eines Sommerkurses von zwei Lehrkräften an einer High-School in Texas durch. Dazu wurde der Unterricht sechs Wochen lang beobachtet. Die Lehrer*innen fertigten täglich Feldnotizen an und sammelten von fünf neu zugewanderte „Fokusschülerinnen“ mit spanischem Sprachhintergrund sämtliche schulische Produkte. Die Ergebnisse erwiesen die Bedeutung davon, Schüler*innen bereits früh in ein wechselseitiges Übersetzungssystem (,language broker“, Hansen-Thomas et al. 2020, S. 161) einzubeziehen, da sie auf diese Weise auch bei noch geringen Englischkenntnissen schnell dem Unterricht folgen können. Die Lehrkräfte bekamen einen besseren Zugang zum fachlichen Wissen der Schüler*innen und deren Englischkenntnissen (Hansen-Thomas et al. 2020, S. 158). Die Schüler*innen fühlten sich aktiviert und involviert und beteiligten sich intensiv am Unterricht. Frustrationen bei den Schüler*innen mit sehr geringen Englischkenntnissen nahmen ab. 
Bauer et al. (2020) verweisen auf die bislang vorliegenden Befunde zur Verbesserung von Wortschatz und sprachlicher Komplexität, mikrostrukturellen Fertigkeiten der Planung des Sprechens und grammatischer Strukturen, höherer Genauigkeit und Vollständigkeit sprachlicher Produktionen als Vorteile gegenüber monolingualem Unterricht. Sie weisen allerdings auch auf Ergebnisse hin, die eine einfache Übernahme des Translanguaging bei mangelnder Berücksichtigung der Unterschiede einbezogener Sprachen problematisieren (Bauer et al. 2020, S. 6f.). Die Autor*innen betonen, dass bislang kaum Studien zu benachteiligten Schüler*innen vorliegen. In ihrer eigenen Studie untersuchen sie dann fallstudienartig sechs Schüler*innen aus Familien mit geringem sozioökonomischem Status einer Schule im Mittelwesten der USA. Die Unterrichtssituation wird beschrieben als kooperatives Lernen in Gruppen mit vielen Kommunikationsanlässen unter den Schüler*innen. Der literacyorientierte Unterricht zeigt Aktivitäten wie das Heraussuchen hochfrequenter Wörter, Lesen, spontane Reaktionen auf Text, Schreiben der Lieblingspassage nach dem Hören einer Geschichte, Reimspiele, Wortjagden. Es wurden mündliche Texte (Erzählen einer Bildergeschichte) und eine Leseaufnahme in Spanisch und Englisch erhoben, transkribiert und ausgewertet. Die Ergebnisse bieten wichtige Einblicke in das Lernen und die Strategien der Schüler*innen wie z. B. Selbstgespräche bei der Suche nach Wörtern oder die Verwendung von Passepartoutwörtern. Die Autor*innen sehen zusammengefasst ein reiches Verständnis von Sprachen, Literalität und dem Erzählen einer Geschichte bei den Schüler*innen, was sie als Ergebnis translingualer Praktiken im Unterricht einordnen (Bauer et al. 2020, S. 23).

Neben den positiven Auswirkungen auf Lernprozesse der Schüler*innen durch den Gebrauch translingualer Praktiken, stellen weitere Studien darüber hinaus die Wirkmächtigkeit gesellschaftlicher Diskurse in Form von Sprachprestige heraus. Sahr (2020) fand die starke Wirkung eines defizitorientierten gesellschaftlichen Diskurses über spanisch-englische Zweisprachigkeit in ihrer Untersuchung zur Verknüpfung von Sprachgebrauch, Sprachidentität und Ideologie. Zwar kommt auch sie in ihrer qualitativen Fallstudie mit Audioaufnahmen des Unterrichts sowie jeweils drei Interviews mit den 19 Teilnehmer*innen zu der Schlussfolgerung, dass Translanguaging zur Sprachvermittlung - in diesem Fall der Fremdsprache Deutsch - positiv beiträgt, aber dass die Möglichkeit, das gesamte sprachliche Repertoire zu nutzen, stark an ethnische Identifizierungen sowie Wissensbestände der bestehenden Communities gebunden ist. Interessant erscheint, dass in dieser Atmosphäre einer hierarchischen Wertigkeit von Sprachen die Fremdsprache - in diesem Fall Deutsch - eine moderierende Rolle in der Öffnung zur Reflexion von Sprachidentitäten gewinnen kann.

Auch in der Studie zum Translanguaging im Englischunterricht von Aoyama (2020) tritt der Effekt des Sprachprestige hervor. Es wurden 190 japanische Schüler*innen in einen Survey zu ihren Sprachpraxen einbezogen und in zehn Klassen jeweils 50 min Videoaufnahmen und mit neun Schüler*innen semistrukturierte Interviews durchgeführt. Beobachtet wurde die Nutzung des Translanguaging in kommunikativen Situationen mit unterschiedlichem Ausmaß bei allen Schüler*innen. Dabei erweist sich der Einsatz des Japanischen primär strategisch motiviert, z. B. zur Überbrückung fehlender sprachlicher Mittel in der Fremdsprache und Unterstützung von Mitschüler*innen bei Sprachschwierigkeiten im Englischen. Davon abgesehen 
blieben die meisten Schüler*innen davon überzeugt, dass die Nutzung des Japanischen im Unterricht für den Erwerb der Fremdsprache kontraproduktiv sei. Die Autor*innen konstatieren eine Diskrepanz zwischen den translingualen Praktiken und den Überzeugungen der Schüler*innen.

Eine ethnographische Studie von Levine und Swanson (2019) in einer Berliner Willkommensklasse für neu zugewanderte Schüler*innen plädiert aufgrund einer Reihe von ethnographischen Aufnahmen - präsentiert als Vignetten - für Translanguaging als Mittel der Anerkennung der sprachlichen Ressourcen und des Abbaus von Hierarchien und für die Zusammenarbeit. Die Zielsetzung ist für die Autor*innen nicht die Erfassung von Wirkungen im Sinne von sprachlichen und fachlichen Leistungen, sondern ein Arrangement des Unterrichts, das sich auch den emotionalen Anforderungen öffnet, nämlich dass Schüler*innen sich wahrgenommen fühlen. Auf dieser Grundlage plädieren sie dafür, Translanguaging in die Curricula für den Unterricht in Vorbereitungsklassen zu integrieren.

Schließlich erlauben Studien im Rahmen hochschulischer Bildung ebenfalls einen Einblick in die Bedingungen der Implementation des Translanguaging: Santoso (2020) führte neben Videoaufnahmen des Unterrichts semistrukturierte Interviews mit fünf Englisch-Studierenden in Jakarta zu ihren Erfahrungen, Gefühlen und Meinungen zum erlebten Unterricht. Als Ergebnis weist sie aus, dass Scaffolding im Unterricht unter Einbezug von Translanguaging funktioniert, um komplexe Begriffe $\mathrm{zu}$ verstehen und Lernprobleme zu lösen, gerade auch durch Unterstützung anderer Lernender. Auch Wang und Curdt-Christiansen (2019) untersuchten den Einsatz von Translanguaging in einem bilingualen Programm für Studierende im Bereich Management in China. Die Studie ist ethnographisch angelegt und verlief über zwei Jahre; es wurden 1500 min Unterricht aufgezeichnet und 36 semistrukturierte Interviews mit Lehrkräften, Administrator*innen und Studierenden durchgeführt sowie Dokumente analysiert. Der Beitrag berichtet verschiedene Typen von Translanguaging-Strategien. Hinsichtlich der Implementationsbedingungen wird die Unterstützung und Gewährung von Freiräumen in der Gestaltung durch die administrativ und politisch Verantwortlichen betont, die die Vorteile des Translanguaging für den Englischerwerb wie auch für das Erlernen der Fachsprache wahrnahmen (Wang und Curdt-Christiansen 2019, S. 331), was von den Studierenden genauso gesehen wurde (Wang und Curdt-Christiansen 2019). Grenzen wurden in Kursen mit komplexen theoretischen Inhalten und einer dichten Fachsprache wie z. B. im Recht gesehen (Wang und Curdt-Christiansen 2019). Die Studierenden kritisierten die Dominanz der Schriftlichkeit und zu geringe Möglichkeiten, Englisch zu sprechen (Wang und Curdt-Christiansen 2019, S. 333). Ein interessanter Effekt ist die sowohl bei den Lehrenden wie den Studierenden gewachsene Toleranz gegenüber der Englischperformanz der anderen.

In den meisten der genannten Studien wird auf die Bedeutung von Fortbildungen für die Verbreitung und Weiterentwicklung des Translanguaging hingewiesen. Neben der o.g. Studie von Panagiotopoulou und Hammel (2020) setzen auch Duarte und Günther-van der Meij (2020) bei der Lehrkräfteprofessionalisierung an und zeigen anhand von Videoaufnahmen sowie vignettenbasierten Diskussionen aus zwei Forschungsprojekten an Schulen in den Niederlanden, wie wichtig es ist, im Rahmen von Fortbildungen die Kompetenz von Lehrkräften in den Dimensionen Können, 
Wissen und Haltungen zu adressieren. Die Autorinnen arbeiten für die Niederlande heraus, dass Lehrkräfte durchaus bereit sind, die Mehrsprachigkeit von Schüler*innen wertzuschätzen und produktiv in den Unterricht miteinzubeziehen (Duarte und Günther-van der Meij 2020, S. 140), dazu aber entsprechende Arbeitsbedingungen fordern. Als eine Voraussetzung dafür werden sichere Räume herausgestellt, in denen sich Lehrkräfte ausprobieren und ihre Erfahrungen mit Kolleg*innen und Wissenschaftler*innen diskutieren können. Wie an anderen Stellen auch wird die Bedeutung wissenschaftlicher Begleitung für die Implementation von Innovationen im Bereich sprachlicher Bildung sichtbar.

Zusammenfassend betrachtet thematisieren die hier aufgeführten Studien entlang verschiedener Etappen der Bildungslaufbahn neben der konkreten Gestaltung des Unterrichtsalltags auch immer wieder die Diskursebene der gesellschaftlichen Akzeptanz von Mehrsprachigkeit. Dabei stellen Fortbildungen sowie Räume für Feedback und Reflexionen eine wichtige Implementationsbedingung dar. Anhand der dargestellten Studien wird deutlich, dass eine rege Forschungslandschaft sowie vielfältige konzeptionelle Überlegungen zum Translanguaging erst im Entstehen sind. Vor dem Hintergrund einer hohen sprachlichen Diversität überrascht das nicht; so bietet Translanguaging ein Konzept, der fluiden und dynamischen sprachlichen Realität mehrsprachiger Schüler*innen zu begegnen und sie als Ressource in den Unterricht einzubeziehen.

Eine vollgültige Studie zur Wirkung translingualer Praktiken im Unterricht und ihrer systematischen Implementation auf die sprachliche und fachliche Entwicklung von Kindern, Jugendlichen, Studierenden oder Erwachsenen mittels der Messung z.B. von Effektstärken in einem Vergleichsgruppendesign steht aus. Fragen der Implementation von Translanguaging werden bislang vorrangig auf der Ebene der Unterrichtsorganisation und der Gestaltung des pädagogischen Alltags im Rahmen didaktischer Forschung behandelt. Deutlich wird zudem auch die Notwendigkeit weiterer Unterstützung, so z. B. durch konkrete Unterrichtsmaterialien zur Unterstützung eines translingualen (Fach-)Unterrichts. Eine Lücke klafft auch hier in der Untersuchung der bildungspolitischen und bildungsadministrativen Ebene. Nach wie vor beschränkt sich das Translanguaging in Bildungsinstitutionen auf einzelne oder in kleinen Zusammenschlüssen organisierte, durch Hochschulen begleitete, Einrichtungen; eine flächendeckende Implementation ist nicht in Sicht. Das Thema ist noch im Stadium der an früherer Stelle genannten ,Innovationsinseln“ (Becker-Mrotzek und Butterworth 2018). Die vorliegenden Ergebnisse legen immerhin eine positive Wirkung auf die Lernatmosphäre und eine stärkere Involvierung von Schüler*innen in sprachliches Lernen wie auch vereinzelt fachliches Lernen nahe.

Wie kann man die hier vorgetragenen Erkenntnisse, Befunde und auch offenen Fragen zusammenfassen?

\section{Zusammenfassung und Ausblick zu Implementationsforschung und Implementation sprachlicher Bildung}

Auf Basis der vorhergehenden Ausführungen zum Stand der Implementationsforschung im Bereich sprachlicher Bildung fassen wir im Folgenden unsere Überlegun- 
gen thesenartig zusammen, wobei sowohl der Stand der Implementation sprachlicher Bildung wie auch die darauf bezogene Forschung berücksichtigt wird.

1. Es gibt kaum Implementationsforschung zu sprachlicher Bildung im engeren Sin$n e$. Die Recherche zum aktuellen Forschungsstand ergab nicht nur keine hinreichende Anzahl von Studien, sondern zeigte insgesamt ein wenig zusammenhängendes Feld, dessen Ergebnisse sich nur schwer in ein Gesamtbild integrieren lassen. Die Gründe dafür sind vielfältig: Von Einfluss ist zum einen die Auswahl der Implementationsgegenstände, wie Trainings von Sprachförderung, Konzepte von insbesondere mehrsprachiger Unterrichtsorganisation wie das Translanguaging oder die Implementation von Minderheitensprachen in Bildungssystemen unterschiedlicher Staaten. Zum anderen handelt es sich um ganz unterschiedliche Zielgruppen bezüglich Alter, sprachlicher Voraussetzungen und Zielvorstellungen, so z. B. wenn es sich um Bildungsangebote für ein- oder mehrsprachige Lerner*innen oder auch für Fremdsprachenlerner*innen handelt. Hinzu kommt, dass sich Schwierigkeiten durch die im Ländervergleich divergierenden Kontextbedingungen insbesondere auf der bildungspolitischen Entscheidungsebene sowie hinsichtlich der Rahmenbedingungen der Bildungssysteme ergeben. Aus den genannten Gründen stützte sich die Darstellung auf die „Implementationsforschung im weiteren Sinne“ (Schrader et al. 2020) und bezog auch Studien ein, in denen Implementationsfragen nicht im zentralen Fokus lagen, aber die Ergebnisse wichtige Aufschlüsse zum Thema anboten.

2. Das lässt sich zusammenfassen: Implementation ist ein komplexes Feld. Grundsätzlich ist von heterogenen Ausgangslagen auszugehen, und das auf ganz unterschiedlichen Ebenen. Einerseits scheint Implementation von Innovationen im Bereich sprachlicher Bildung genau von dieser Heterogenität zu leben und aus diesen sehr unterschiedlichen Bedingungen, Erfahrungen, Kompetenzen nicht nur Herausforderungen für die technische Seite der Umsetzung zu produzieren, sondern auch eine kreative Gestaltung hinsichtlich Organisation und Durchführung zu ermöglichen. Andererseits ist es notwendig, dieses komplexe Feld zusammenzubringen und zusammenzuhalten; dazu dienen gemeinsame Instrumente, Routinen und Standards, schließlich auch eine gemeinsam getragene Überzeugung von der Richtigkeit des eigenen Tuns, eine Identifizierung. Es geht also nicht nur um die möglichst konzepttreue Adaption einer sprachlichen Bildungsmaßnahme, sondern ebenfalls um abgestimmte Prozesse, Kommunikation, Entscheidungsfindung, Steuerung usw., die eine gemeinsame Orientierung der Pädagog*innen gewährleisten und gleichzeitig als Ressourcen für die Verständigung und Entwicklung von kreativen Lösungen zur Verfügung stehen.

3. Weiterhin beginnt Implementation nicht erst mit der konkreten Umsetzung einer sprachbildnerischen Maßnahme mit Kindern und Jugendlichen, sondern schon früh im Stadium der Entscheidung. Implementation ist ein Prozess und kein $\mathrm{Zu}$ stand. Es bedarf also einer längerfristigen Zeitplanung, beginnend mit einer Vorbereitungsphase, in der es Gelegenheiten für Erprobungen gibt. Es folgt die Programminstallation und eine initiale Umsetzung. Selbst bei bereits empirisch gut abgesicherten Maßnahmen wird es Überarbeitungsphasen geben, die sich auf die Art und Weise beziehen, wie sie organisiert werden, bevor es zur vollen Umset- 
zung kommt. In dieser Zeit geht es um Korrektur und Nachsteuerung, bis die Verankerung im System erreicht ist. Eine Entscheidung zum Transferbedarf i.d.R. einer übergeordneten Entscheidungsfindung.

4. Reine Top-down- oder Bottom-up-Steuerung lässt sich in den Studien kaum ausmachen. Adaptive Implementation ist der Normalfall. In der Regel verlangt Implementation eine Balancierung der Steuerung. Das mag zum einen an der langjährigen Strategie der Autonomisierung von Institutionen im Bildungswesen wie auch am Selbstbild vieler Pädagog*innen als ,reflective practitioners“ (Schön 1983) liegen. Zum anderen ist das der heterogenen Ausgangslage in der Praxis vor Ort geschuldet. Zur Bewältigung dessen hat eine partizipatorische Anlage von Implementationen als Modell gerade in den bundesweiten Programmen zur sprachlichen Bildung eine größere Bedeutung gewonnen.

5. Es geht schließlich darum, Menschen davon zu überzeugen, etwas Neues auszuprobieren und den ihnen gewohnten Praxisalltag zu verändern, manchmal auch auf den Kopf zu stellen. Implementation ist für die einzelnen Akteur*innen eine Bewegung und eine Anstrengung: Das betrifft nicht nur neue fachliche Anforderungen und Kompetenzen, sondern auch die Ebene der sozialen Organisation. Die Implementation neuer Konzepte sprachlicher Bildung verlangt einen gemeinsamen Prozess, gerade auch wenn systematische Verbesserungen für jene Gruppen angestrebt sind, die ohne Unterstützung der Schule keine Chance auf eine zukunftsfähige Bildung haben. Von daher braucht Implementation im hier vorgeschlagenen Sinne Kooperation, Kommunikation, Austausch, gemeinsame Reflexion bis hin zu gemeinsamer Arbeit, zum Beispiel im Teamteaching. Ebenso verlangt Implementation, nicht nur mit Kolleg*innen zusammenzuarbeiten, sondern sich in vielen Fällen auch auf andere Instanzen einzulassen wie zum Beispiel Wissenschaftler*innen, Multiplikator*innen u.a.m.

6. Für die Bedingungen der Implementation sprachlicher Bildung liegen inzwischen viele einzelne Ergebnisse vor, die auf der Mikro- und Mesoebene die wesentlichen Faktoren erfassen, nämlich solche, die in den Personen der Lehrkräfte bzw. pädagogischen Fachkräfte liegen, sowie strukturierende Elemente wie die Bedeutung von Konzepttreue, Adaption, Machbarkeit und Steuerung. Weniger Erkenntnisse liegen zur übergeordneten Entscheidungsebene der Bildungspolitik vor. Als ein zentraler Bereich können Maßnahmen der Qualifizierung und Professionalisierung gelten. Die vorliegenden Studien liefern einen durchaus reichhaltigen Einblick in die Bedeutung von Fortbildungen und ihrer Gestaltung hinsichtlich der Formate und methodischen Elemente. Studien mit einem übergreifenden Ansatz, die es erlauben die Wirkweisen der einzelnen Elemente in ihrem Zueinander (zum Beispiel als Pfadmodelle) zu modellieren und zu erfassen, stehen aus. Das gilt für die schulische Bildung und die Elementarbildung. Allerdings lässt sich ein Bemühen erkennen, kausale Zusammenhänge stärker in den Blick zu bekommen.

7. Stark abstrahiert lässt sich sagen, dass die Studien zur sprachlichen Bildung ohne oder mit nur geringer Berücksichtigung der Mehrsprachigkeit vor allem Wirkfaktoren auf der Mikro- und Meso-Ebene, also dem Feld der Einrichtungen selbst, berichten, wohingegen Studien, die eine mehrsprachige Bildung untersuchen, auch über Einflussfaktoren auf der Makroebene im bildungspolitischen Feld berichten. An dieser Stelle soll hervorgehoben werden, dass insbesondere für das wohl inter- 
national bekannteste Modell einer mehrsprachigen Bildung - die bilinguale twoway immersion oder auch: Dual Language Education - hinsichtlich seiner Implementationsbedingungen mit einer gezielten Recherche zu Implementation und Transfer nur wenige Beiträge gefunden wurden, die, zusammen betrachtet, kein systematisches Bild zum aktuellen Stand ergeben. Vielleicht ist es symptomatisch, dass sich ein neuerer Übersichtsartikel von Lindholm-Leary (2018) in einem Sammelband geradezu versteckt. Deshalb erfolgte im vorliegenden Beitrag eine Schwerpunktsetzung auf das Translanguaging. Denn nur für dieses sprachdidaktische Konzept, das mehrsprachige Bildung nicht auf zwei Unterrichtssprachen begrenzt, was in bilingualen Modellen zu einer gewissen Künstlichkeit hinsichtlich der Organisation und Zusammensetzung von Lerngruppen führt, ließ sich überhaupt eine nennenswerte Anzahl verwertbarer Studien finden. Translanguaging bietet ein Innovationspotenzial, das in den letzten Jahren auch in Deutschland vermehrt Aufmerksamkeit erhält.

8. Für die herangezogenen Studien besteht angesichts großer Unterschiede der methodischen Anlagen eine begrenzte Vergleichbarkeit. Diese Unterschiede ergeben sich auch aus den verschiedenen Erkenntnisinteressen: Als Interventions- und Evaluationsstudien bedienen sich die Studien aus den bundesweiten Programmen mehrheitlich standardisierter Methoden und greifen dabei teilweise auch auf Längsschnitt- und Kontrollgruppendesigns zurück. Häufig setzen sie dabei quantitative und qualitative Methoden in Kombination ein, folgen also einem Mixed-Methods-Ansatz. Befragungen von großen Gruppen von Schüler*innenund Lehrkräften mit Hilfe von Tests und Kompetenzmessungen stehen im Mittelpunkt und werden durch Fragebögen, Beobachtungen und semistrukturierte Interviews ergänzt (siehe die Übersichtstab. 1 im Anhang). Anders als bei den bundesweiten Programmen, die spezifische Sprachförderprogramme und deren Wirkung untersuchen, zielen die Studien zum Translanguaging stärker darauf, Erfahrungen zu sammeln und Erkenntnisse zu generieren, um auf deren Basis eine mehrsprachige Didaktik zu entwickeln; sie arbeiten daher eher konzeptionell. Die meisten Studien dazu zielen auf ein Verständnis und die Exploration des Phänomens $\mathrm{ab}$, sie nutzen eher selten standardisierte, sondern bevorzugen offenere qualitative Designs, hauptsächlich (semistrukturierte) Interviews, aber auch Beobachtungen und Unterrichtsaufnahmen. Sie konzentrieren sich auf Fallstudien und eine kleinere Zahl von Befragten. Bezüglich der Untersuchungseinheiten fällt auf, dass die Studien aus den bundesweiten Programmen häufig sowohl die Lehrkräfte oder die pädagogischen Fachkräfte als auch die Kinder und Jugendlichen in den Blick nehmen, während sich die meisten Translanguaging-Studien auf eine der beiden Gruppen konzentrieren. Die Studien aus den verschiedenen Bereichen folgen damit mehrheitlich unterschiedlichen Forschungslogiken, was die Vergleichbarkeit der Ergebnisse einschränkt und eher eine gegenseitige Ergänzung als eine Aggregation der Erkenntnisse erlaubt.

Der hier vorgelegte Überblick zur Implementationsforschung im Bereich sprachlicher Bildung unter besonderer Berücksichtigung von Mehrsprachigkeit ist als ein Tableau zu verstehen. Die Gruppierung der einbezogenen Studien zeigt nicht nur eine große thematische Bandbreite, sondern auch unterschiedliche Formen der for- 
schungsmethodischen Zugänge. Das gewonnene Bild müsste weiter komplettiert werden, zeigt aber auch Felder auf, zu denen sichere Befunde vorliegen. Es wird deutlich, dass im Feld der sprachlichen Bildung in den letzten Jahren Implementationsfragen stärker in den Vordergrund gerückt sind und zu den Gelingensbedingungen der sprachlichen Bildung vorgestoßen wurde. Allerdings stehen auf die Verkehrssprache und auf Mehrsprachigkeit gerichtete Konzepte nach wie vor weitgehend nebeneinander. 


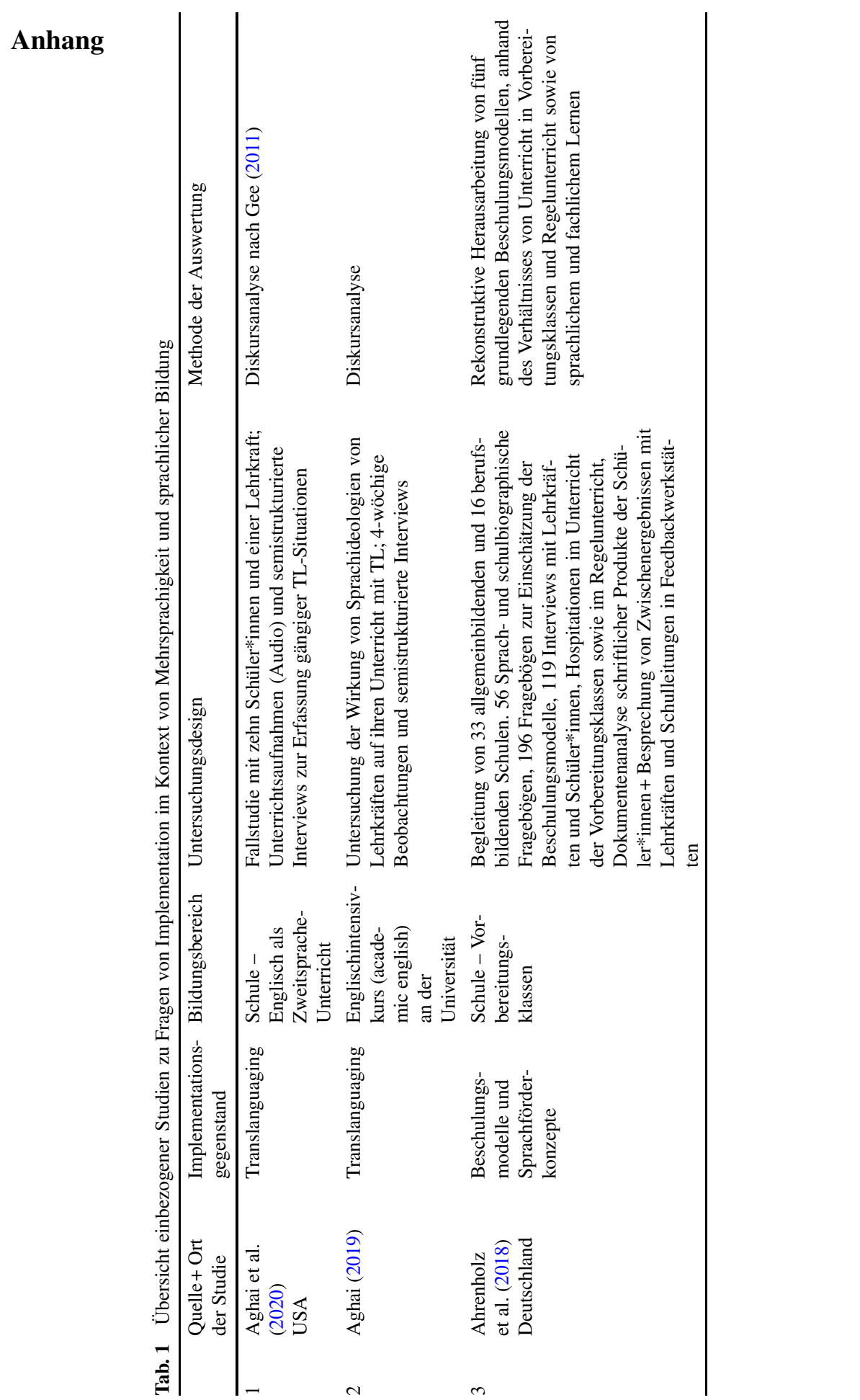




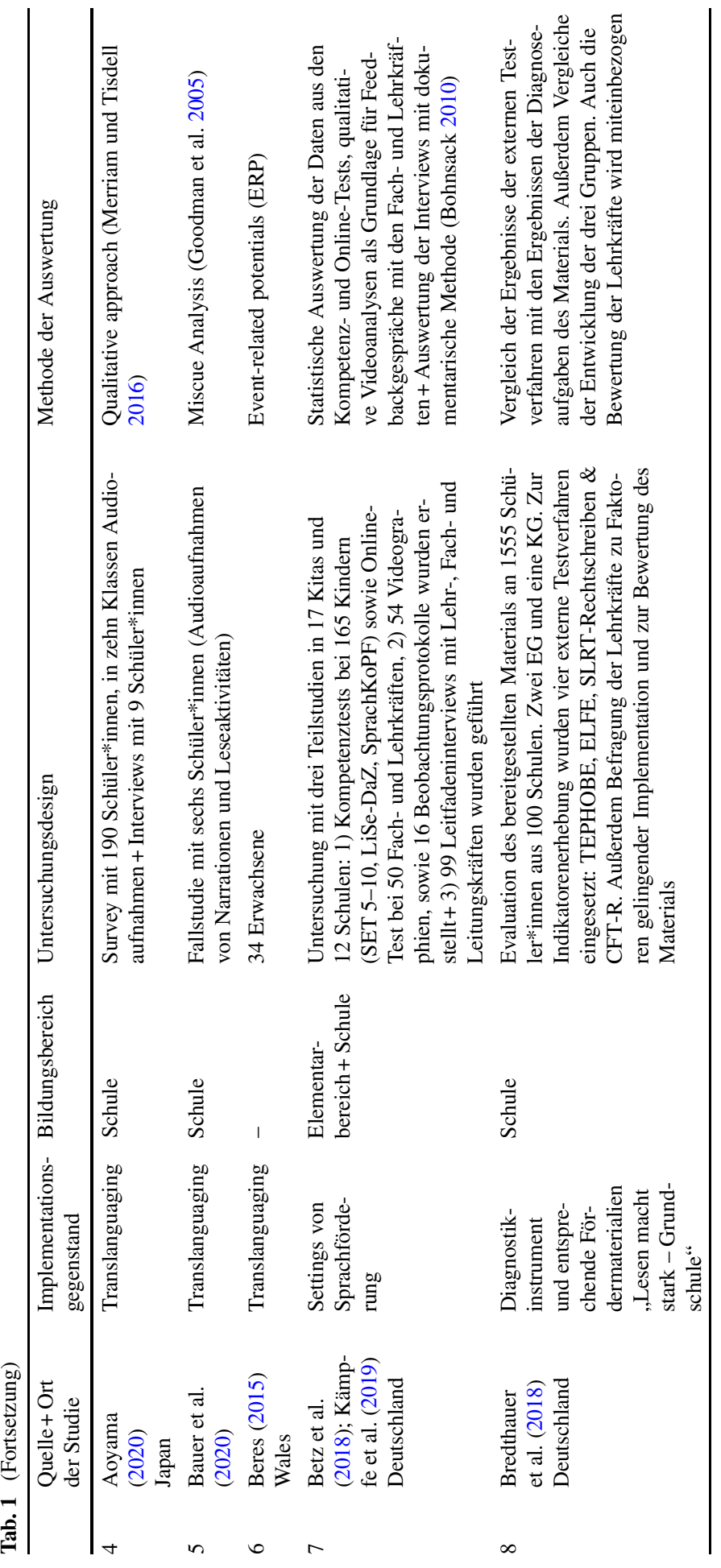




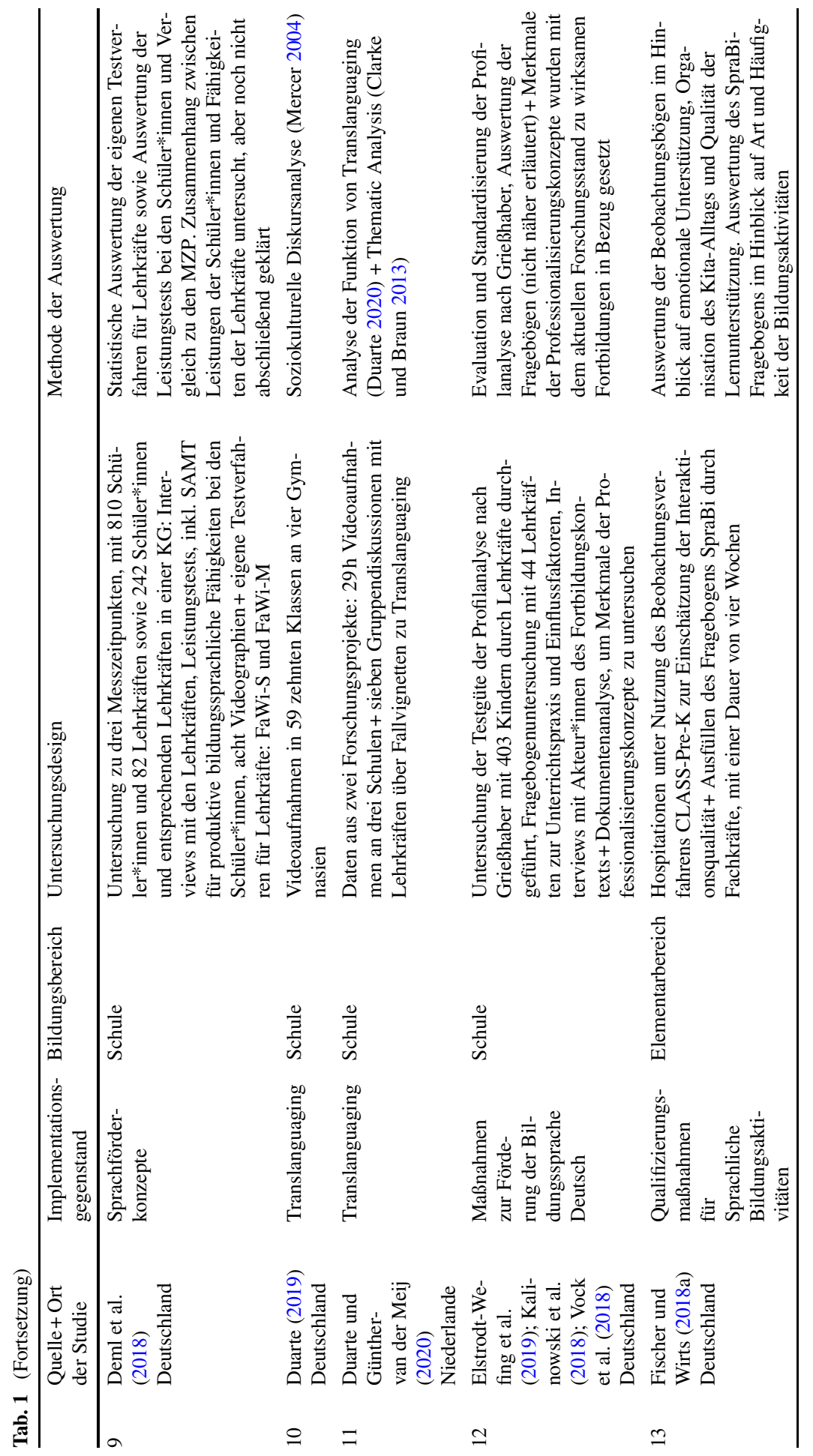




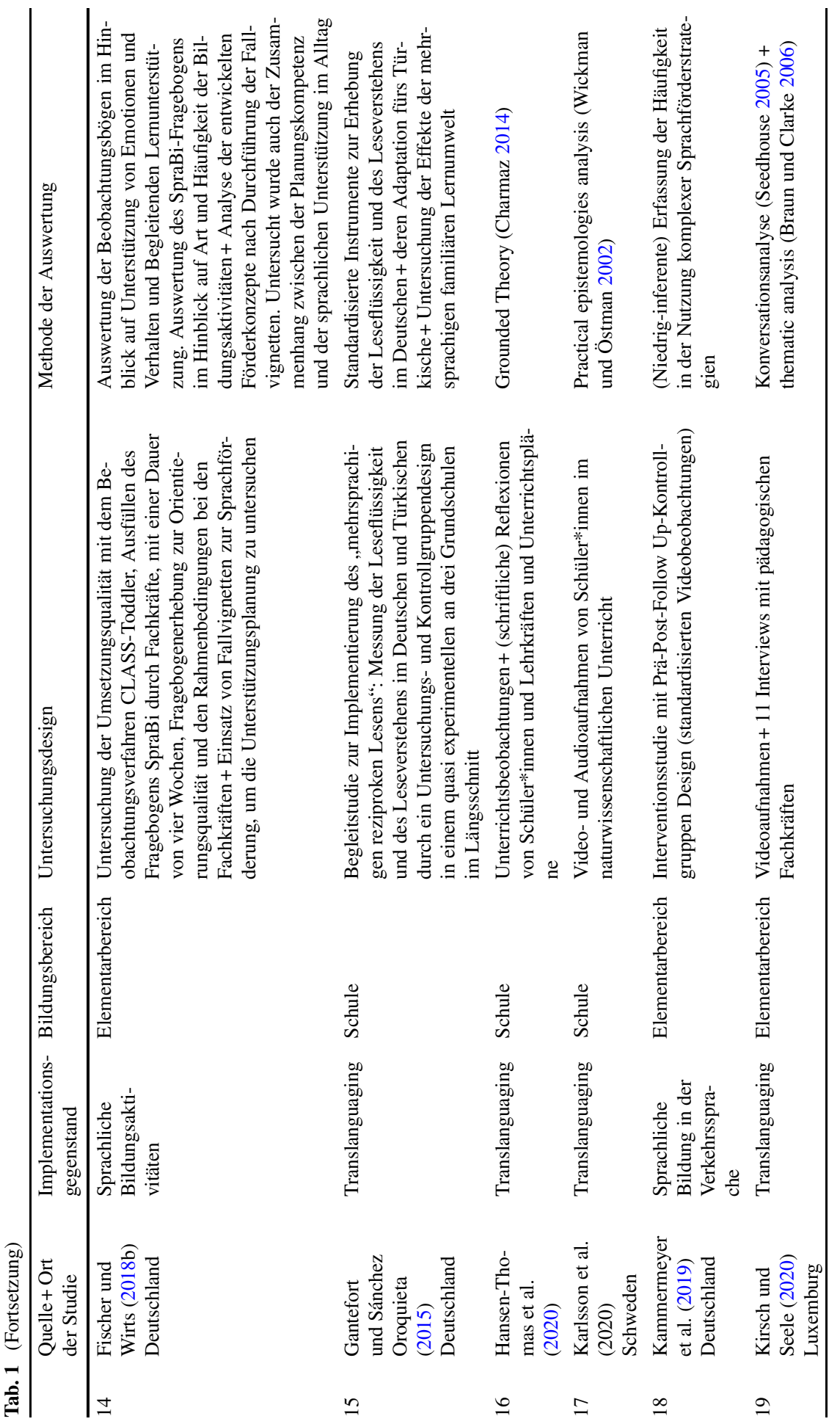




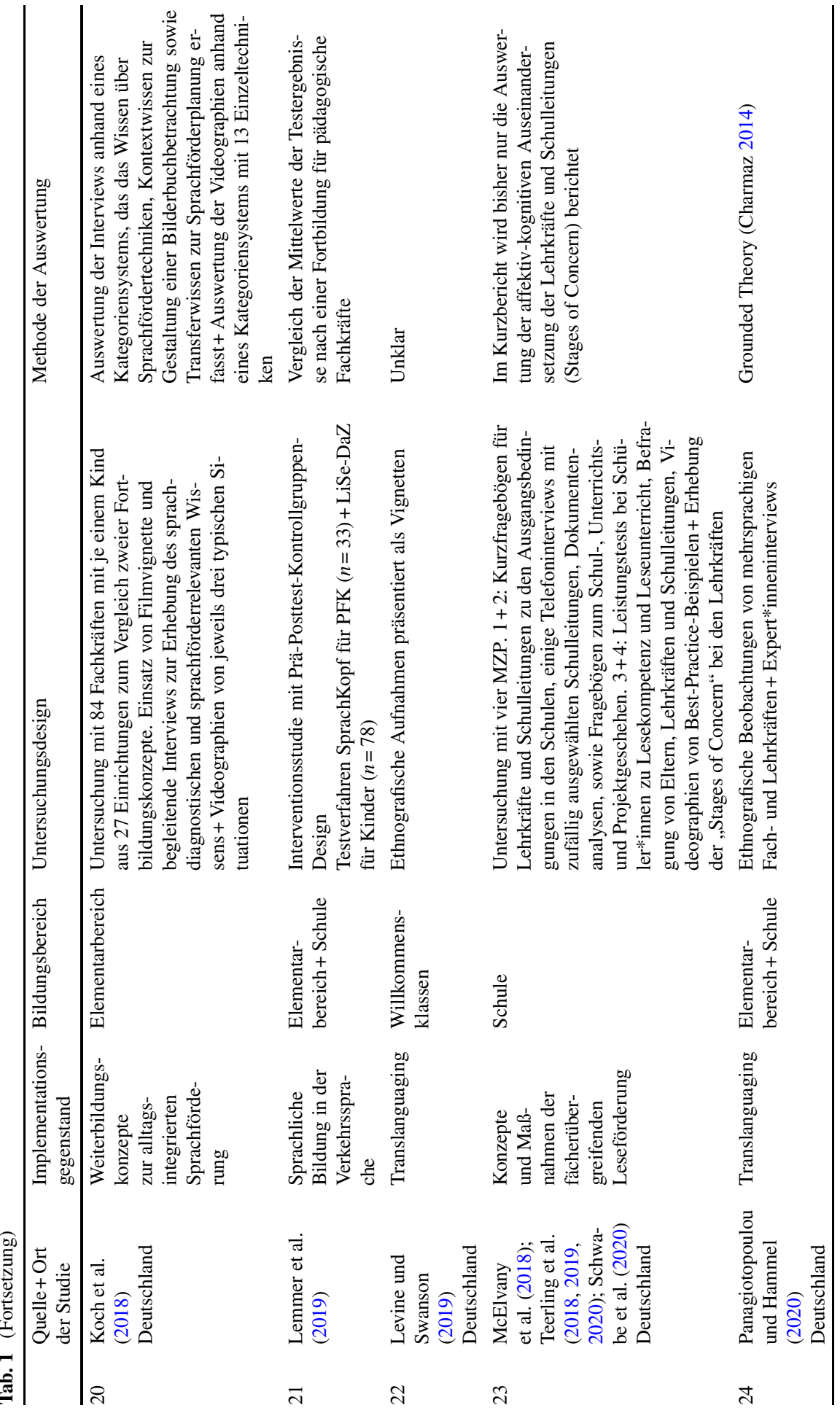




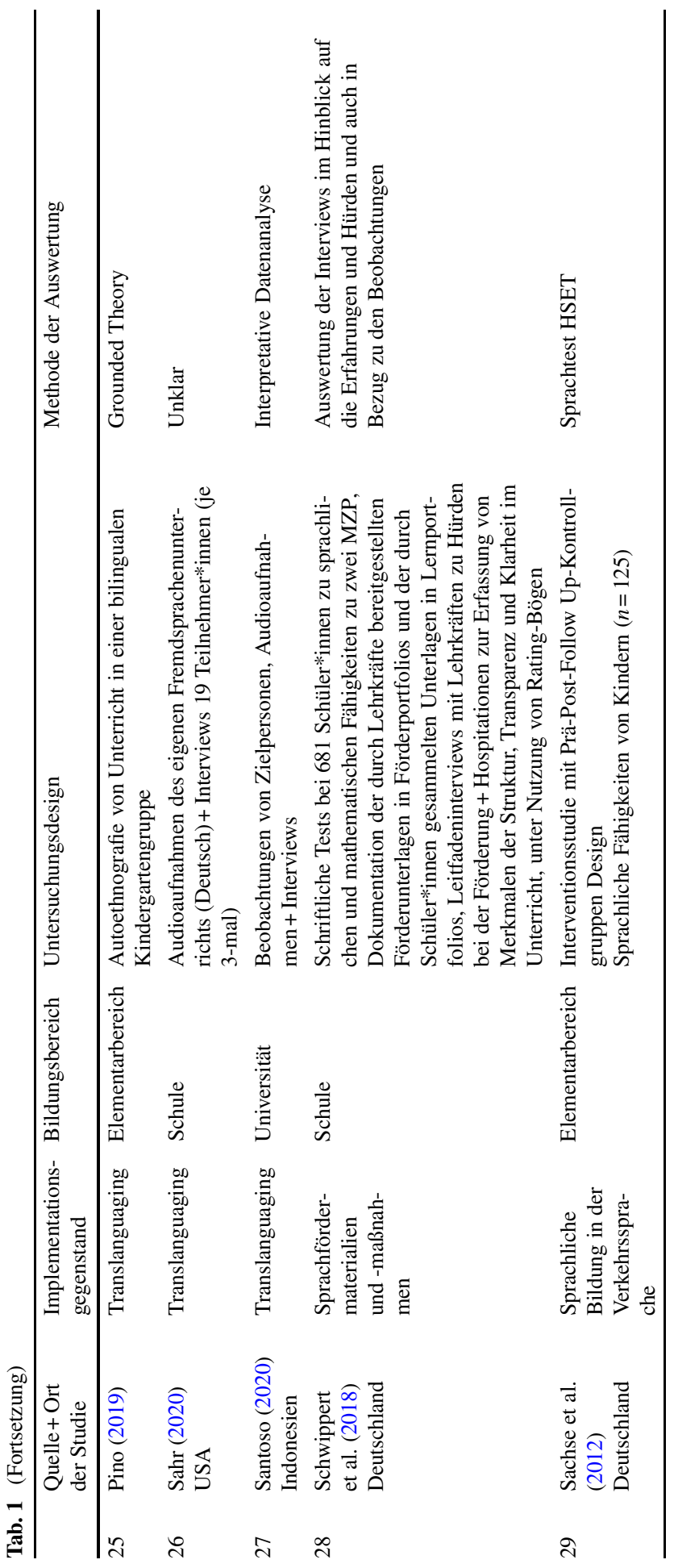




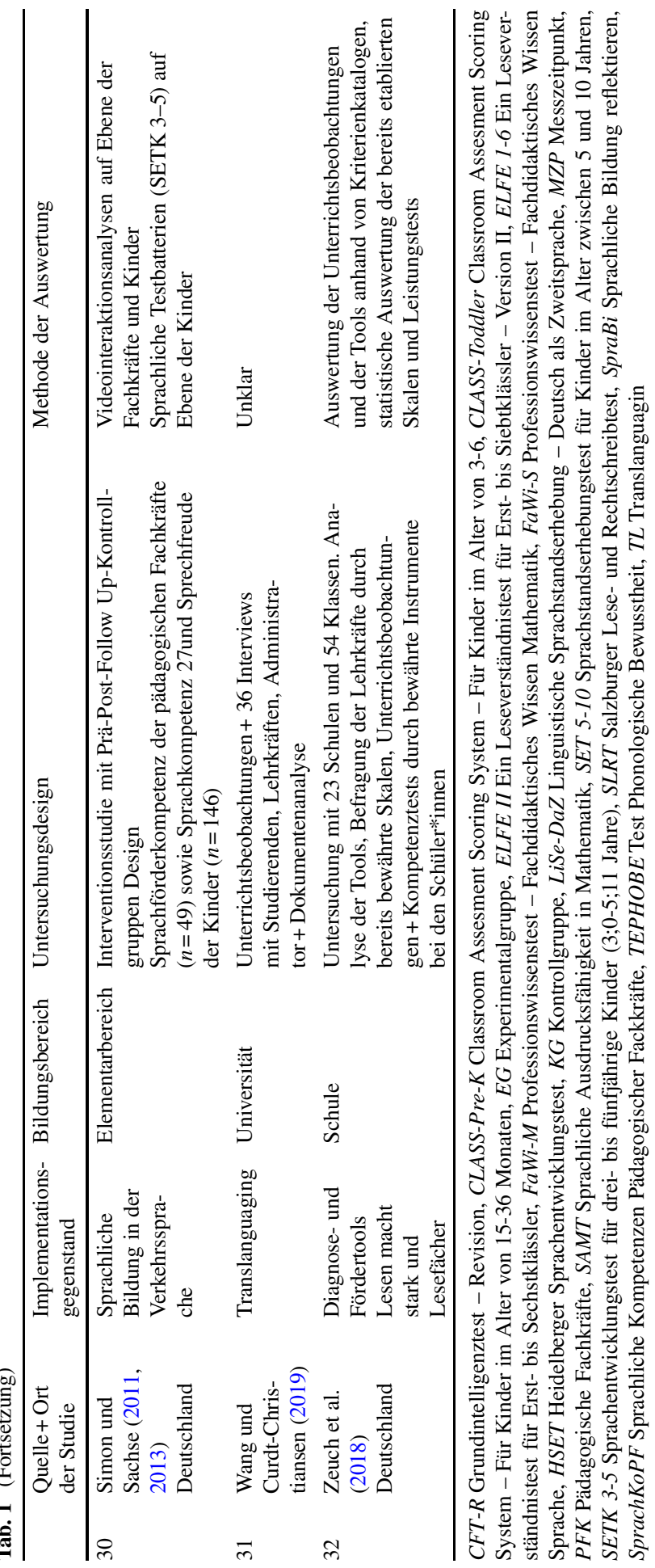


Funding Open Access funding enabled and organized by Projekt DEAL.

Open Access Dieser Artikel wird unter der Creative Commons Namensnennung 4.0 International Lizenz veröffentlicht, welche die Nutzung, Vervielfältigung, Bearbeitung, Verbreitung und Wiedergabe in jeglichem Medium und Format erlaubt, sofern Sie den/die ursprünglichen Autor(en) und die Quelle ordnungsgemäß nennen, einen Link zur Creative Commons Lizenz beifügen und angeben, ob Änderungen vorgenommen wurden.

Die in diesem Artikel enthaltenen Bilder und sonstiges Drittmaterial unterliegen ebenfalls der genannten Creative Commons Lizenz, sofern sich aus der Abbildungslegende nichts anderes ergibt. Sofern das betreffende Material nicht unter der genannten Creative Commons Lizenz steht und die betreffende Handlung nicht nach gesetzlichen Vorschriften erlaubt ist, ist für die oben aufgeführten Weiterverwendungen des Materials die Einwilligung des jeweiligen Rechteinhabers einzuholen.

Weitere Details zur Lizenz entnehmen Sie bitte der Lizenzinformation auf http://creativecommons.org/ licenses/by/4.0/deed.de.

\section{Literatur}

Aghai, L. (2019). What teachers need to know about English language learners' translanguaging in the classroom. In J. Kengwe \& G. Onchwari (Hrsg.), Handbook of research on assessment practices and pedagogical models for immigrant students (S. 109-126). Hershey: IGI Global.

Aghai, L., Sayer, P., \& Vercellotti, M.L. (2020). Effects of teachers' language ideologies on language learners' translanguaging practices in an intensive English program. In Z. Tian, L. Aghai, P. Sayer \& J.L. Schissel (Hrsg.), Envisioning TESOL through a Translanguaging lens. Global perspectives (S. 345-362). Cham: Springer.

Ahrenholz, B., Birnbaum, T., Ohm, U., \& Brede, R. J. (2018). EVA-Sek. Formative Prozessevaluation in der Sekundarstufe. Seiteneinsteiger und Sprache im Fach. In S. Henschel, S. Gentrup, L. Beck \& P. Stanat (Hrsg.), Projektatlas Evaluation. Erste Ergebnisse aus den BiSS-Evaluationsprojekten (S. 43-47). Berlin: BiSS-Trägerkonsortium.

Aoyama, R. (2020). Exploring Japanese high school students' L1 use in translanguaging in the communicative EFL classroom. TESL-EJ, 23(4), 1-18.

Bauer, E. B., Colomer, S.E., \& Wiemelt, J. (2020). Biliteracy of African American and Latinx Kindergarten students in a dual-language program: understanding students' Translanguaging practices across informal assessments. Urban Education, 55(3), 331-361.

Becker-Mrotzek, M., \& Butterworth, J. (2018). Qualitätsbestimmende Faktoren bei der Implementierung von (Sprach-)Förderkonzepten. In C. Titz, S. Weber, A. Ropeter, S. Geyer \& M. Hasselhorn (Hrsg.), Konzepte zur Sprach- und Schriftsprachförderung umsetzen und überprüfen (S. 46-64). Stuttgart: Kohlhammer.

Bellmann, J., \& Müller, T. (2011). Evidenzbasierte Pädagogik - ein Déjà-vu? Einleitende Bemerkungen zur Kritik eines Paradigmas. In J. Bellmann \& T. Müller (Hrsg.), Wissen, was wirkt (S. 9-32). Wiesbaden: VS.

Benson, C. (2004). Bilingual schooling in Mozambique and Bolivia: from experimentation to implementation. Language Policy, 3(1), 47-66.

Beres, M. (2015). Translanguaging as a strategy to boost human learning: An event-related potential $(E R P)$ investigation. Doctoral dissertation. Wales: Bangor University.

Betz, T., Erdogan, E., Kämpfe, K., Kucharz, D., Mehlem, U., \& Rezagholinia, S. (2018). SPRÜNGE. Sprachförderung im Übergang Kindergarten-Grundschule evaluieren. In S. Henschel, S. Gentrup, L. Beck \& P. Stanat (Hrsg.), Projektatlas Evaluation. Erste Ergebnisse aus den BiSS-Evaluationsprojekten (S. 26-29). Berlin: BiSS-Trägerkonsortium.

Bohnsack, R. (2010). Rekonstruktive Sozialforschung. Einführung in qualitative Methoden (8. Aufl.). Opladen: Barbara Budrich.

Bredthauer, S., Jambor-Fahlen, S., Prien, A., Scholz, V., \& Weiß, J. (2018). Niemanden zurücklassen Lesen macht stark - Grundschule - Diagnose und Förderung von Lese- und Schreibkompetenzen im Primarbereich. In C. Titz, S. Weber, H. Wagner, A. Ropeter, S. Geyer \& M. Hasselhorn (Hrsg.), 
Konzepte zur Sprach- und Schriftsprachförderung umsetzen und überprüfen (S. 128-145). Stuttgart: Kohlhammer.

Braun, V., \& Clarke, V. (2006). Using thematic analysis in psychology. Qualitative Research in Psychology, $3(2), 77-101$.

Brügelmann, H. (2019). „Zu Risiken und Nebenwirkungen...“. Warnung vor einer naiven „Evidenzbasierung“" in der Pädagogik. Lehren und lernen, 7(5), 29-34.

Charmaz, K. (2014). Constructing Grounded Theory (2. Aufl.). London: Sage Publications.

Clarke, V., \& Braun, V. (Hrsg.). (2013). Successful qualitative research: A practical guide for beginners. London: Sage.

Deml, I., Binder, K., Schulte, M., Merkert, A., Bien-Miller, L., Wildemann, A., Schilcher, A., Krauss, S., Lenske, G., \& Rank, A. (2018). Eva-Prim. Evaluation im Primarbereich: Sprachförderung in alltäglichen und fachlichen Kontexten im Rahmen der Bund-Länder-Initiative BiSS. In S. Henschel, S. Gentrup, L. Beck \& P. Stanat (Hrsg.), Projektatlas Evaluation. Erste Ergebnisse aus den BiSSEvaluationsprojekten (S. 32-34). Berlin: BiSS-Trägerkonsortium.

Duarte, J. (2011). Bilingual language proficiency. A comparative study. Münster: Waxmann.

Duarte, J. (2019). Translanguaging in mainstream education: a sociocultural approach. International Journal of Bilingual Education and Bilingualism, 22(2), 150-164.

Duarte, J. (2020). Translanguaging in the context of mainstream multilingual education. International Journal of Multilingualism, 17(2), 232-247.

Duarte, J., \& Günther-van der Meij, M.T. (2020). 'we learn together'-translanguaging within a holistic approach towards Multilingualism in education. In J. A. Panagiotopoulou, L. Rosen \& J. Strzykala (Hrsg.), Inclusion, education and translanguaging. How to promote social justice in (teacher) education? (S. 125-144). Wiesbaden: Springer VS.

Egert, F., Galuschka, K., Groth, K., Hasselhorn, M., \& Sachse, S. (2020). Evidenzbasierung vorschulischer sprachlicher Bildung und Förderung: Was man darunter versteht und bisher darüber weiß. In K. Blatter, K. Groth \& M. Hasselhorn (Hrsg.), Evidenzbasierte Überprüfung von Sprachförderkonzepten im Elementarbereich (S. 3-27). Wiesbaden: Springer VS.

Elstrodt-Wefing, N., Starke, A., Möhring, M., \& Ritterfeld, U. (2019). Umsetzung unterrichtsintegrierter Sprachförderung im Primarbereich. Eine Mixed-Method-Untersuchung bei Lehrkräften in BiSSVerbünden. Empirische Sonderpädagogik, 3, 191-209.

Fischer, S., \& Wirts, C. (2018a). BiSS-E1. Alltagsintegrierte Sprachbildung und -diagnostik in Kitas: Formative Prozessevaluation der Bund-Länder-Initiative „Bildung durch Sprache und Schrift“. In S. Henschel, S. Gentrup, L. Beck \& P. Stanat (Hrsg.), Projektatlas Evaluation. Erste Ergebnisse aus den BiSS-Evaluationsprojekten (S. 18-21). Berlin: BiSS-Trägerkonsortium.

Fischer, S., \& Wirts, C. (2018b). BiSS-E2. Alltagsintegrierte Sprachbildung und -diagnostik in Kitas: Formative Prozessevaluation der Bund-Länder-Initiative „Bildung durch Sprache und Schrift“. In S. Henschel, S. Gentrup, L. Beck \& P. Stanat (Hrsg.), Projektatlas Evaluation. Erste Ergebnisse aus den BiSS-Evaluationsprojekten (S. 14-17). Berlin: BiSS-Trägerkonsortium.

Fixsen, D. L., Naoom, S. F., Blasé, K. A., Friedman, R. M., \& Wallace, F. (2005). Implementation research: a synthesis of the literature. Tampa: University of South Florida, Louis de la Parte Florida Mental Health Institute, The National Implementation Research Network.

Fürstenau, S. (2016). Multilingualism and School Development in Transnational Educational Spaces. Insights from an Intervention Study at German Elementary Schools. In A. Küppers, B. Pusch \& P. UyanSemerci (Hrsg.), Bildung in transnationalen Räumen (S. 71-90). Wiesbaden: Springer VS.

Fürstenau, S., Çelik, Y., \& Plöger, S. (2020). Language Comparison as an Inclusive Translanguaging Strategy: Analysis of a Multilingual Teaching Situation in a German Primary School Classroom. In J. A. Panagiotopoulou, L. Rosen \& J. Strzykala (Hrsg.), Inclusion, Education and Translanguaging. How to Promote Social Justice in (Teacher) Education? (S. 145-162). Wiesbaden: Springer VS.

Gantefort, C. (2013). Schriftliches Erzählen mehrsprachiger Kinder. Entwicklung und sprachenübergreifende Fähigkeiten. Münster: Waxmann.

Gantefort, C., \& Sánchez Oroquieta, M. J. (2015). Translanguaging-Strategien im Sachunterricht der Primarstufe: Förderung des Leseverstehens auf Basis der Gesamtsprachigkeit. Transfer Forschung $\leftrightarrow$ Schule, 1(1), 24-37.

Gantefort, C., \& Maahs, I.-M. (2020). Translanguaging. Mehrsprachige Kompetenzen von Lernenden im Unterricht aktivieren und wertschätzen. https://www.uni-due.de/imperia/md/content/prodaz/ gantefort_maahs_translanguaging.pdf. Zugegriffen: 15. Okt. 2020.

García, O., \& Kleifgen, J.A. (2020). Translanguaging and literacies. Reading Research Quarterly, 55(4), 553-571. 
García, O., \& Lin, M. Y. (2016). Translanguaging in bilingual education. In O. García, M. Y. Lin \& S. May (Hrsg.), Bilingual and multilingual education (S. 117-130). Cham: Springer.

Gee, J. P. (2011). How to do discourse analysis: A toolkit (2. Aufl.). New York, NY: Routledge.

Gogolin, I. (1997). „Arrangements“ als Hindernis \& Potential für Veränderung der schulischen sprachlichen Bildung. In I. Gogolin \& U. Neumann (Hrsg.), Großstadt-Grundschule. Eine Fallstudie über sprachliche und kulturelle Pluralität als Bedingung der Grundschularbeit (S. 311-345). Münster: Waxmann.

Gogolin, I., \& Neumann, U. (Hrsg.). (2009). Streitfall Zweisprachigkeit - The Bilingualism Controversy. Wiesbaden: VS.

Gogolin, I., \& Roth, H.-J. (2007). Bilinguale Grundschule: Ein Beitrag zur Förderung der Mehrsprachigkeit. In T. Anstatt (Hrsg.), Mehrsprachigkeit bei Kindern und Erwachsenen. Erwerb - Formen Förderung (S. 31-46). Tübingen: Narr Francke Attempto Verlag.

Gogolin, I., Dirim, I., Klinger, T., Lanke, I., Drorit, L., Michel, U., Neumann, U., Reich, H. H., Roth, H.J., \& Schwippert, K. (2011). Förderung von Kindern und Jugendlichen mit Migrationshintergrund FörMig. Bilanz und Perspektiven eines Modellprogramms. Münster: Waxmann.

Gogolin, I., Neumann, U., \& Roth, H.-J. (2007). Bericht 2007. Abschlussbericht über die italienisch-deutschen, portugiesisch-deutschen und spanisch-deutschen Modellklassen. (unter Mitarbeit von A. Grevé und T. Klinger). Hamburg: mimeo.

Gomolla, M., Schwendowius, D., \& Kollender, E. (2016). Qualitätsentwicklung von Schulen in der Einwanderungsgesellschaft: Evaluation der Lehrerfortbildung zur interkulturellen Koordination (2012-2014). https://edoc.sub.uni-hamburg.de/hsu/volltexte/2016/3139/pdf/Evaluation_Qualitaets entwicklung_von_Schulen_in_der_Einwanderungsgesellschaft.pdf. Zugegriffen: 15. Okt. 2020. (Hamburger Beiträge zur Erziehungs- und Sozialwissenschaft, Heft 16). Hamburg Helmut-Schmidt Universität/Universität der Bundeswehr.

Goodman, Y., Watson, D., \& Burke, C. (2005). Reading miscue inventory. Katonah, NY: Richard C. Owen Publishers.

Gräsel, C. (2010). Stichwort: Transfer und Transferforschung im Bildungsbereich. Zeitschrift für Erziehungswissenschaft, 13(1), 7-20.

Gräsel, C., \& Parchmann, I. (2004). Implementationsforschung - oder: der steinige Weg, Unterricht zu verändern. Unterrichtswissenschaft, 32(3), 196-214.

Groth, K., Egert, F., \& Sachse, S. (2017). Wirksamkeit eines additiven Sprachförderkonzepts für mehrsprachige Kinder. Frühe Bildung, 6(2), 74-82.

Hansen-Thomas, H., Stewart, M., Flint, P., \& Dollar, T. (2020). Co-learning in the high school English class through translanguaging: emergent bilingual newcomers and monolingual teachers. Journal of Language, Identity \& Education, 20(3), 151-166.

Hartmann, U., Ehm, J., Höltge, L., \& Hasselhorn, M. (2020). Unter welchen Bedingungen gelingt die Implementation eines Modellvorhabens zur Förderung der Schulbereitschaft in Kindertagesstätten? Zeitschrift für Erziehungswissenschaft, 23, 61-82.

Hasselhorn, M., Köller, O., Maaz, K., \& Zimmer, K. (2014). Implementation wirksamer Handlungskonzepte im Bildungsbereich als Forschungsaufgabe. Psychologische Rundschau, 65(3), 140-149.

Henschel, S., Stanat, P., Hasselhorn, M., Becker-Mrotzek, M., \& Roth, H.-J. (2014). Evaluationskonzept der Bund-Länder-Initiative „Bildung durch Sprache und Schrift“. https://www.biss-sprachbildung. de/pdf/Evaluationskonzept-BiSS.pdf. Zugegriffen: 15. Okt. 2020.

Kalinowski, E., Gronostaj, A., Westphal, A., \& Vock, M. (2018). Lehrkräftefortbildung und Sprachförderung - Eine Tagebuchstudie im Projekt „Bildung durch Sprache und Schrift“. Empirische Pädagogik, 32(2), 216-232.

Kammermeyer, G., King, S., Goebel, P., Lämmerhirt, A., Leber, A., Papillion-Piller, A., \& Metz, A. (2017). Mit Kindern im Gespräch. Strategien zur Sprachbildung und Sprachförderung von Kindern in Kindertageseinrichtungen. Donauwörth: Auer.

Kammermeyer, G., Metz, A., Leber, A., Roux, S., Biskup-Ackermann, B., \& Fondel, E. (2019). Wie wirken sich Weiterbildungen auf die Anwendung von Sprachförderstrategien von pädagogischen Fachkräften in Kitas aus? Frühe Bildung, 8(4), 212-222.

Kämpfe, K., Betz, T., Kucharz, D., \& Rezagholinia, S. (2019). Gemeinsame Sprachförderung am Übergang Kita-Grundschule: Handlungsorientierungen und Sprachförderkompetenzen von Fach- und Lehrkräften. Frühe Bildung, 8(4), 206-211.

Karlsson, A., Larsson, P. N., \& Jakobsson, A. (2020). The continuity of learning in a translanguaging science classroom. Cultural Studies of Science Education, 15, 1-25.

Kirsch, C., \& Seele, C. (2020). Translanguaging in early childhood education in Luxembourg: from practice to pedagogy. In J.A. Panagiotopoulou, L. Rosen \& J. Strzykala (Hrsg.), Inclusion, education 
and translanguaging. How to promote social justice in (teacher) education? (S. 63-81). Wiesbaden: Springer VS.

Koch, K., v. Dapper-Saalfels, T., Machowiak, K., Beckerle, C., Löffler, C., Pauer, I., \& Heil, J. (2018). allE. Gelingsbedingungen alltagsintegrierter sprachlicher Bildung im Elementarbereich. In S. Henschel, S. Gentrup, L. Beck \& P. Stanat (Hrsg.), Projektatlas Evaluation. Erste Ergebnisse aus den BiSSEvaluationsprojekten (S. 22-25). Berlin: BiSS-Trägerkonsortium.

Kratzmann, J., Jahreiss, S., Frank, M., Ertanir, B., \& Sachse, S. (2017). Einstellungen pädagogischer Fachkräfte in Kindertageseinrichtungen zur Mehrsprachigkeit. Zeitschrift für Erziehungswissenschaft, 20(2), 237-258.

Lemmer, R., Huschka, S., Geyer, S., Brandenburg, J., Ehm, J.-H., Lausecker, A., Schulz, P., \& Hasselhorn, M. (2019). Sind Fortbildungsmaßnahmen zu linguistisch fundierter Sprachförderung wirksam? - Analysen zu den Kompetenzen von Fachkräften und mehrsprachigen Kindern. Frühe Bildung, 8(4), $181-186$.

Lengyel, D., Ilić, V., Rybarski, K., \& Schmitz, M. (2019). Evaluation „Rucksack Schule“ im Kreis Unna. Kurzzusammenfassung der zentralen Ergebnisse. https://kommunale-integrationszentrennrw.de/sites/default/files/public/system/downloads/kurzzusammenfassung_erkenntnisse_evaluation_ rucksack_schule_lengyel_u.a.pdf. Zugegriffen: 15. Okt. 2020.

Leutner, D. (2013). Perspektiven pädagogischer Interventionsforschung. Bielefeld: Bertelsmann.

Levine, G., \& Swanson, B. (2019). Fostering compassion through translanguaging pedagogy in the German Willkommensklasse. Language Education and Multilingualism-The Langscape Journal, 2, $167-179$.

Lewis, G., Jones, B., \& Baker, C. (2012). Translanguaging: origins and development from school to street and beyond. Educational Research and Evaluation, 18(7), 641-654.

Lindholm-Leary, K. (2012). Success and challenges in dual language education. Theory Into Practice, 5(4), 256-262.

Lindholm-Leary, K. (2018). Implications of research for dual language at the early childhood level. In M. B. Arias \& M. Fee (Hrsg.), Profiles of dual language education in the 21st century (S. 73-85). Bristol: Multilingual Matters.

McElvany, N., Köller, O., Ohle-Peters, A., Igler, I., Schlitter, T., \& Asseburg, R. (2018). Evaluation der Leseförderung im Rahmen von „Bildung durch Sprache und Schrift (BiSS)“. In F. Schwabe, N. McElvany, W. Bos \& H. G. Holtappels (Hrsg.), Schule und Unterricht in gesellschaftlicher Heterogenität (Jahrbuch der Schulentwicklung, Bd. 20, S. 258-280). Weinheim: Beltz Juventa.

Mercer, N. (2004). Sociocultural discourse analysis: analysing classroom talk as a social mode of thinking. Journal of Applied Linguistics, 1(2), 137-168

Merriam, S., \& Tisdell, E. (2016). Qualitative research: A guide to design and implementation (4. Aufl.). San Francisco: Jossey-Bass.

Meyers, D.C., Durlak, J.A., \& Wandersman, A. (2012). The quality implementation framework: a synthesis of critical steps in the implementation process. American Journal of Community Psychology, $50(3 / 4), 462-480$.

Möller, J., Hohenstein, F., Fleckenstein, J., Köller, O., \& Baumert, J. (2016). Erfolgreich integrieren - die Staatliche Europa-Schule Berlin. Münster: Waxmann.

Neumann, U., \& Roth, H.-J. (2004). Bilinguale Grundschulklassen in Hamburg - Ein Werkstattbericht. Grenzgänge. Zeitschrift für eine moderne Romanistik, 21, 31-60.

Neumann, U., \& Roth, H.-J. (2009). Multilingual primary schools in Germany-models and research. In M.E. Torres-Guzman \& J. Gòmez (Hrsg.), Global perspectives on multilinguals. Unity in diversity (S. 85-105). New York: Teachers College Columbia University.

Neurath, O. (1981). Protokollsätze. In R. Haller \& R. Rutte (Hrsg.), Gesammelte philosophische und methodologische Schriften (S. 577-585). Wien: Hölder-Pichler-Tempsky. Zuerst in: Erkenntnis, 3, 204-214.

Otheguy, R., García, O., \& Reid, W. (2015). Clarifying translanguaging and deconstructing named languages: a perspective from linguistics. Applied Linguistics Review, 6(3), 281-307.

Panagiotopoulou, A. (2016). Mehrsprachigkeit in der Kindheit. Perspektiven für die frühpädagogische Praxis. München: Deutsches Jugendinstitut e. V.

Panagiotopoulou, J.A., \& Hammel, M. J. (2020). 'what shall we sing now, amir?' developing a voice through translanguaging pedagogy. An ethnographic research and professional training project in day-care centers and schools. In J. A. Panagiotopoulou, L. Rosen \& J. Strzykala (Hrsg.), Inclusion, education and translanguaging. How to promote social justice in (teacher) education? (S. 203-218). Wiesbaden: Springer VS. 
Panagiotopoulou, J.A. (2018). Inklusion und Mehrsprachigkeit. Translanguaging in Kitas und Schulen. zmI-Magazin, 2018(12), 11-13.

Pino, Y. (2019). Translanguaging in a Kindergarten classroom: freedom to teach and learn. New York: Hofsta University.

Proctor, E. K., Landsverk, J., Aarons, G., \& Chambers, D. (2009). Implementation research in mental health services: an emerging science with conceptual, methodological, and training challenges. $A d$ ministration and Policy in mental health and Mental Health Services Research, 36(1), 24-34.

Reich, H.H., \& Roth, H.-J. (2002). Spracherwerb zweisprachig aufwachsender Kinder und Jugendlicher. Ein Überblick über den Stand der nationalen und internationalen Forschung. Hamburg: Behörde für Bildung und Sport. http://www.hamburg.de/contentblob/69654/data/bbs-hr-spracherwerbzweisprachigkeit-11-02.pdf. Zugegriffen: 15. Okt. 2020.

Rolff, H.-G. (2019). Transfer von Innovationen im Schulbereich. In C. Schreiner, C. Wiesner, S. Breit, P. Dobbelstein, M. Heinrich \& U. Steffens (Hrsg.), Praxistransfer Schul- und Unterrichtsentwicklung (S. 49-60). Münster: Waxmann.

Roth, H.-J., \& Terhart, H. (Hrsg.). (2015). Rucksack. Empirische Befunde und theoretische Einordnungen zu einem Elternbildungsprogramm für mehrsprachige Familien. Münster: Waxmann.

Sachse, S., Budde, N., Rinker, T., \& Groth, K. (2012). Evaluation einer Sprachfördermaßnahme für Vorschulkinder. Frühe Bildung, 1(4), 194-201.

Sahr, A. (2020). Translanguaging practices of multilingual learners of German. Athens Journal of Education, 7, 49-76.

Santoso, W. (2020). Translanguaging through the lens of sociocultural approach: students' attitudes and practices. Journal Pendidikan Bahasa, 9(1), 1-19.

Schneider, W., Baumert, J., Becker-Mrotzek, M., Hasselhorn, M., Kammermeyer, G., Rauschenbach, T., Roßbach, H.-G., Roth, H.-J., Rothweiler, M., \& Stanat, P. (Hrsg.). (2012). Expertise „Bildung durch Sprache und Schrift (BiSS)“: Bund-Länder-Initiative zur Sprachförderung, Sprachdiagnostik und Leseförderung. http://www.biss-sprachbildung.de/ueber-biss/biss-expertise. Zugegriffen: 15. Okt. 2020.

Schön, D. (1983). The reflective practicioner: How professionals think in action. New York: Basic Books.

Schrader, J., Hasselhorn, M., Hetfleisch, P., \& Goeze, A. (2020). Stichwortbeitrag Implementationsforschung: Wie Wissenschaft zu Verbesserungen im Bildungssystem beitragen kann. Zeitschrift für Erziehungswissenschaft, 23(1), 9-59.

Schüler-Meyer, A., Prediger, S., Wagner, J., \& Weinert, H. (2018). Bedingungen für zweisprachige Lernangebote. Videobasierte Analysen zu Nutzung und Wirksamkeit einer Förderung zu Brüchen. Psychologie in Erziehung und Unterricht, 66, 161-175.

Schwippert, K., Neumann, A., Leiss, D., Groß, N., Entrich, S., Plath, J., \& Weber, A. (2018). EvaFa. Evaluation der Sprachförderung im Fachunterricht der Sekundarstufe I im BiSS-Programm. In S. Henschel, S. Gentrup, L. Beck \& P. Stanat (Hrsg.), Projektatlas Evaluation. Erste Ergebnisse aus den BiSS-Evaluationsprojekten (S. 39-42). Berlin: BiSS-Trägerkonsortium.

Seedhouse, P. (2005). Conversation Analysis and language learning. Language Teaching, 38(4), $165-187$.

Simon, S., \& Sachse, S. (2011). Sprachförderung in der Kindertagesstätte. Verbessert ein Interaktionstraining das sprachförderliche Verhalten von Erzieherinnen? Empirische Pädagogik, 25(4), 462-480.

Simon, S., \& Sachse, S. (2013). Anregung der Sprachentwicklung durch ein Interaktionstraining für Erzieherinnen. Diskurs Kindheits- und Jugendforschung/Discourse. Journal of Childhood and Adolescence Research, 8(4), 379-397.

Souvignier, E., \& Philipp, M. (2016). Implementation - Begrifflichkeiten, Befunde und Herausforderungen. In M. Philipp \& M. Souvignier (Hrsg.), Implementation von Lesefördermaßnahmen (S. 9-22). Münster: Waxmann.

Stevenson, A. R. (2013). How fifth grade Latino/a bilingual students use their linguistic resources in the classroom and laboratory during science instruction. Cultural Studies of Science Education, 8(4), 973-989.

Teerling, A., Asseburg, R., Igler, J., Schlitter, T., Ohle-Peters, A., Köller, O., \& McElvany, N. (2018). BiSS-EvalLesen. Bildung durch Sprache und Schrift - Evaluation von Konzepten und Maßnahmen der fachübergreifenden Leseförderung im Primarbereich. In S. Henschel, S. Gentrup, L. Beck \& P. Stanat (Hrsg.), Projektatlas Evaluation. Erste Ergebnisse aus den BiSS-Evaluationsprojekten (S. 50-53). Berlin: BiSS-Trägerkonsortium.

Teerling, A., Bernholt, A., Asseburg, R., Hasl, A., Igler, J., Schlitter, T., Ohle-Peters, A., McElvany, N., \& Köller, O. (2019). Affektiv-kognitive Auseinandersetzung mit einer Innovation im Implementationsprozess - Eine modellbasierte Erfassung. Psychologie in Erziehung und Unterricht, 65, 1-18. Reprint Online. 
Teerling, A., Bernholt, A., Igler, J., Schlitter, T., Ohle-Peters, A., McElvany, N., \& Köller, O. (2020). The attitude does matter: the role of principals' and teachers' concerns in an implementation process. International Journal of Educational Research, 100, 1-15.

Titz, C., Weber, S., Ropeter, A., Geyer, S., \& Hasselhorn, M. (Hrsg.). (2018). Konzepte zur Sprach- und Schriftsprachförderung umsetzen und überprüfen. Stuttgart: Kohlhammer.

Vock, M., Gronostaj, A., Grosche, M., Ritterfeld, U., Zaruba, N., Kalinowski, E., Ehl, B., Paul, M., Elstrodt, N., Möhring, M., \& Starke, A. (2018). BiSS-EOS. Förderung der Bildungssprache Deutsch in der Primarstufe: Evaluation, Optimierung und Standardisierung von Tools im BiSS-Projekt. In S. Henschel, S. Gentrup, L. Beck \& P. Stanat (Hrsg.), Projektatlas Evaluation. Erste Ergebnisse aus den BiSS-Evaluationsprojekten (S. 35-38). Berlin: BiSS-Trägerkonsortium.

Wang, W., \& Curdt-Christiansen, X.L. (2019). Translanguaging in a Chinese-English bilingual education programme: a university-classroom ethnography. International Journal of Bilingual Education and Bilingualism, 22(3), 322-337.

Wei, L. (2018). Translanguaging as a practical theory of language. Applied Linguistics, 39(1), 9-30.

Wickman, P., \& Östman, L. (2002). Learning as discourse change: A sociocultural mechanism. Science Education, 86(5), 601-623.

Wong, K. M., \& Benson, C. (2019). Policy and practice in L1-based multilingual education: challenges and steps forward. Fire: Forum for International Research in Education, 5(3), 1-11.

Zeuch, N., Schmitz, A., Karstens, F., Meudt, S.-I., Souvignier, E., \& Jost, J. (2018). EILe. Evaluation der Implementation von Konzepten zur Leseförderung in der Sekundarstufe. In S. Henschel, S. Gentrup, L. Beck \& P. Stanat (Hrsg.), Projektatlas Evaluation. Erste Ergebnisse aus den BiSS-Evaluationsprojekten (S. 54-57). Berlin: BiSS-Trägerkonsortium.

Zydatiß, W. (2000). Bilingualer Unterricht in der Grundschule. Entwurf eines Spracherwerbkonzepts für zweisprachige Immersionsprogramme. Ismaning: Hueber. 